\title{
Flavour structure of R-violating neutralino decays at the LHC
}

\author{
Nils-Erik Bomark, ${ }^{a}$ Debajyoti Choudhury, ${ }^{b}$ Smaragda Lola ${ }^{c, d}$ and Per Osland ${ }^{a}$ \\ ${ }^{a}$ Department of Physics, University of Bergen, \\ Postboks 7803, N-5020 Bergen, Norway \\ ${ }^{b}$ Department of Physics and Astrophysics, University of Delhi, \\ Delhi 11000\%, India \\ ${ }^{c}$ TH Division, Physics Department, CERN, \\ 1211 Geneva 23, Switzerland \\ ${ }^{d}$ Department of Physics, University of Patras, \\ GR-26500 Patras, Greece \\ E-mail: Nils-Erik.Bomark@ift.uib.no, debajyoti.choudhury@gmail.com, \\ Magda.Lola@cern.ch, Per.Osland@ift.uib.no
}

\begin{abstract}
We study signatures of R-parity violation in the production of supersymmetric particles at the LHC, and the subsequent decay of the lightest neutralino being the end product of a supersymmetric cascade decay. In doing so, we pay particular attention to the possible flavour structure of the operators, and how one may discriminate between different possibilities. A neutralino LSP would couple to all quarks and leptons and a comparative study of its decays provides an optimal channel for the simultaneous study of all 45 R-violating operators. By studying the expected signals from all these operators, we demonstrate the ability to understand whether more than one coupling dominates, and to map the experimental signatures to operator hierarchies that can then be compared against theoretical models of flavour. Detailed comparisons with backgrounds, including those from MSSM cascade decays are made, using the PYTHIA event simulator.
\end{abstract}

KEYwORds: Supersymmetry Phenomenology

ARXiv EPRINT: 1105.4022 


\section{Contents}

1 Introduction $\quad 2$

2 Framework for the analysis $\quad 3$

2.1 Cascade dominance 6

$\begin{array}{lll}3 & L L \bar{E} \text { couplings } & 7\end{array}$

3.1 Final-state flavour correlations $\quad 8$

$\begin{array}{lll}3.2 & p_{T} \text { distributions } & 9\end{array}$

3.3 Invariant mass distributions 9

$\begin{array}{ll}3.4 \text { Backgrounds and uncertainties } & 10\end{array}$

3.5 Comparison of Monte Carlo results with theory 11

$\begin{array}{lll}3.6 & \text { Some counting observables } & 14\end{array}$

$\begin{array}{lll}3.7 & \text { Redefining taus } & 19\end{array}$

$\begin{array}{ll}3.8 & \text { Combinations of couplings — case study } 20\end{array}$

$4 \quad L Q \bar{D}$ coupling $\quad 22$

4.1 Event selection $\quad 22$

4.2 Invariant mass distributions 24

$\begin{array}{ll}4.3 & \text { Mixing } L Q \bar{D} \text { operators }\end{array}$

$\begin{array}{lll}4.4 & \text { Backgrounds } & 28\end{array}$

$5 \quad \bar{U} \bar{D} \bar{D}$ coupling $\quad 29$

$6 \quad$ Mixing and models $\quad 31$

6.1 $L L \bar{E}$ and $L Q \bar{D}$ operators $\quad 31$

6.2 Link with flavour symmetries: two examples 32

6.2.1 Left-right-symmetric models 33

$\begin{array}{lll}6.2 .2 & \mathrm{SU}(5) & 36\end{array}$

$\begin{array}{lll}7 & \text { Summary } & 38\end{array}$

$\begin{array}{ll}\text { A Energy spectra from three-body decays } & 40\end{array}$

B Theoretical invariant mass distributions 41 


\section{Introduction}

Over the last decades, there has been increasing interest in R-violating supersymmetry, as a plausible alternative to the Minimal Supersymmetric Standard Model (MSSM). Supersymmetrizing the Standard Model (SM) does allow for additional terms in the superpotential over and above those corresponding to the Higgs potential or the Yukawa terms within the SM. The supersymmetric versions of the latter read

$$
\mu H_{1} H_{2}+m_{e} L_{i} \bar{E}_{j} H_{1}+m_{d} Q_{i} \bar{D}_{j} H_{1}+m_{u} Q_{i} \bar{E}_{j} H_{2},
$$

where $H_{1,2}$ are the Higgs superfields, $L(Q)$ the left-handed lepton (quark) doublet superfields, and $\bar{E}(\bar{D}, \bar{U})$ the corresponding left-handed singlet fields. Gauge symmetry as well as supersymmetry do allow for additional bilinear terms of the form

$$
\mu_{i} L_{i} H_{2}
$$

as well as trilinear ones, namely

$$
\lambda_{i j k} L_{i} L_{j} \bar{E}_{k}+\lambda_{i j k}^{\prime} L_{i} Q_{j} \bar{D}_{k}+\lambda_{i j k}^{\prime \prime} \bar{U}_{i} \bar{D}_{j} \bar{D}_{k} .
$$

Each of the terms in eqs. (1.2) \& (1.3) violate R-parity. In particular, the existence of any of $\mu_{i}, \lambda_{i j k}, \lambda_{i j k}^{\prime}$ implies lepton number violation while a non-zero $\lambda_{i j k}^{\prime \prime}$ violates baryon number. $\mathrm{SU}(2)$ and $\mathrm{SU}(3)$ invariance imply that there are $48 \mathrm{R}$-violating couplings in all $\left(3 \mu_{i}\right.$ 's, $27 \lambda_{i j k}^{\prime}$ s and 9 each of $\lambda_{i j k}$ and $\left.\lambda_{i j k}^{\prime \prime}\right)$.

The strictest bounds on lepton and baryon-number violating operators come from proton stability. The assumption of a conserved R-parity automatically rules out all of the terms of eqs. (1.2) \& (1.3), rendering the proton stable [1, 2], modulo higher dimensional terms endemic to the MSSM. However, alternative symmetries, namely baryon or lepton parities [3-5] can also exclude the simultaneous presence of dangerous $L Q \bar{D}$ and $\bar{U} \bar{D} \bar{D}$ couplings [6]. Experimental constraints from the non-observation of modifications to Standard Model rates, or from possible exotic processes $[7-14,18]^{1}$ also impose additional limits. Overall, the phenomenology to be expected out of such theories is very rich, since the LSP (Lightest Supersymmetric Particle) is no longer stable and the missing-energy signatures of the MSSM $^{2}$ are substituted by multi-lepton or multi-jet events $[25,26] ;^{34}$ single superparticle productions [28-31] are also possible.

In addition to the consequences for collider searches, R-violation implies that gravitinos (which may have been thermally produced after a period of inflation) are also unstable. However, gravitino dark matter in the framework of R-violating supersymmetry is plausible [32-34], provided that the gravitino decays slowly enough for its lifetime to be larger than the age of the universe $[35,36]$. This is an exciting possibility that allows for supersymmetric dark matter, even if the R-violating couplings are sufficiently large to lead to observable signatures at colliders $[33,34]$. Moreover, it was found that the branching ratios for gravitino decays are very sensitive to the flavour structure of R-violating operators [33, 34].

\footnotetext{
${ }^{1}$ For a review of experimental constraints on R-violating operators, see refs. [15-17].

${ }^{2}$ For an introduction to the phenomenology of the MSSM, see refs. [19, 20].

${ }^{3}$ For some of the earliest references on the phenomenology of R-violating supersymmetry, see refs. [21-24].

${ }^{4}$ For a review on R-violating supersymmetry, see [27].
} 
In general, the flavour structure of R-violating couplings is of particular relevance in defining the nature of the signals to be expected and any information on it would be crucial for understanding the flavour structure of the fundamental theory. Indeed, one may try to relate hierarchies amongst $\mathrm{R}$-violating couplings to those in fermion masses [37-42], using models with family symmetries. For example, a large class of such models allow only the third generation fermions to be massive, while the remaining masses are generated by the spontaneous breaking of the family symmetry. In such a scheme, if $\mathrm{R}$ parity is violated, couplings with different family charges are likely to appear with different powers of the family symmetry-breaking parameter, and thus with different magnitudes.

Testing the above models against observations is very hard, since by allowing the most general family structure of couplings one ends up with a large number of possibilities. Single superparticle productions can lead to spectacular signatures, but they are very dependent on the flavour structure and one must ensure that the initial scattering states couple to the operators of interest in a specific model. If this is not the case, the respective single superparticle production mechanisms will be invisible.

From this point of view, pair production of superpartners and their subsequent cascade decays via an unstable neutralino LSP have a great advantage, since the latter (by coupling to all quarks and leptons) could decay via any of the 45 trilinear operators, thereby allowing a comparative study. Through a detailed, correlated study of these decay chains, one may also investigate whether more than one R-violating couplings are of substantial size, "map" their magnitudes and hierarchies, and compare against theoretical models. This is the aim of the present paper, which is structured as follows: in section 2, we summarise the generic framework of the analysis. In section 3, we study energy and invariant mass distributions, for $L L \bar{E}$ operators, as well as leptonic R-violating branching fractions. In sections 4 and 5 we discuss energy and invariant mass distributions for $L Q \bar{D}$ and $\bar{U} \bar{D} \bar{D}$ operators, respectively. In section 6 we focus on the correlations between different couplings and possible mappings between the kinematic distributions and operator flavour hierarchies. In section 7 we present our conclusions. Finally, in appendices A and B we present spectra from three-body decays and theoretical invariant mass distributions, respectively, integrating over the unobserved neutrino energy.

\section{Framework for the analysis}

We study possible effects of R-parity violation (RPV) in the end stages of cascade decays of supersymmetric particles. In other words, we assume that the RPV-couplings are small enough not to materially change either the production processes (which continue to be gauge-interaction driven), nor in the cascade decays down to the neutralino LSP. The LSP, though, decays, within the detector, on account of a non-zero RPV-coupling. A neutralino LSP is motivated even in the case of R-violating supersymmetry, since, due to the lack of electromagnetic or colour interactions, its mass after renormalisation group runs tends to be smaller than those of other sparticles for a very broad region of the supersymmetric parameter space. A sneutrino or a stau LSP (due to the large Yukawa coupling, staus can 


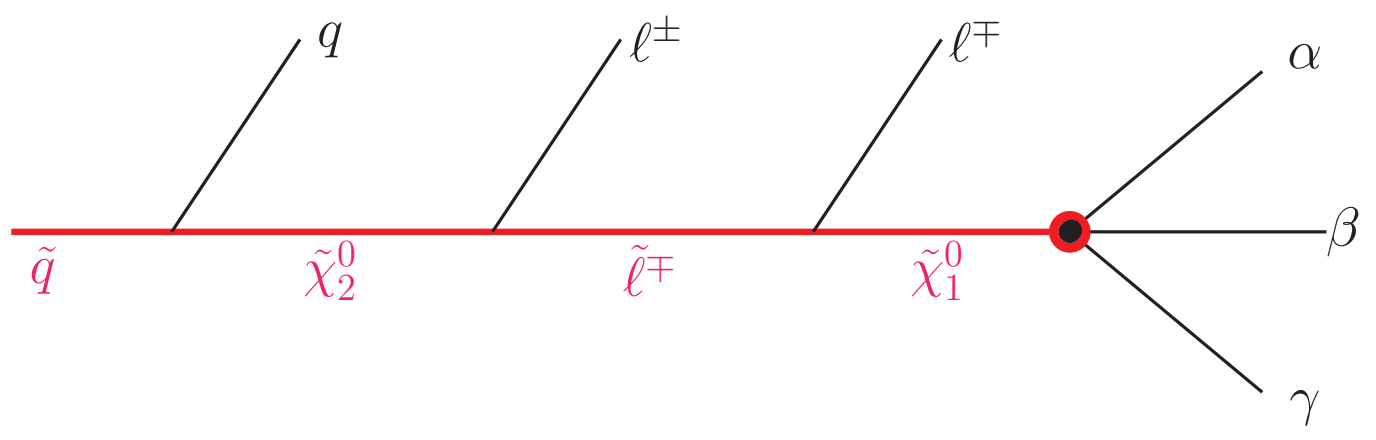

Figure 1. Feynman diagram describing one important supersymmetric decay chain followed by an R-parity violating neutralino decay.

be anomalously light) is still plausible, especially if $m_{0}$ is sufficiently small compared to $m_{1 / 2}$. Those scenarios are beyond the scope of this work.

Depending on the scenario, a variety of supersymmetric particles could be produced in proton-proton collisions, mostly squarks and gluinos,

$$
q q \rightarrow \tilde{q} \tilde{q}, \quad q g \rightarrow \tilde{q} \tilde{g}, \quad g g \rightarrow \tilde{g} \tilde{g}, \quad g g \rightarrow \tilde{q} \tilde{\bar{q}}, \quad q \bar{q} \rightarrow \tilde{q} \tilde{\bar{q}},
$$

but also charginos and neutralinos. The squarks and gluinos will typically decay to quarks and leptons, one important channel being

$$
\begin{aligned}
& \tilde{q} \rightarrow q \tilde{\chi}_{2}^{0} \rightarrow q \ell^{+} \tilde{\ell}^{-} \rightarrow q \ell^{+} \ell^{-} \tilde{\chi}_{1}^{0}, \\
& \tilde{g} \rightarrow \bar{q} \tilde{q} \rightarrow \bar{q} q \tilde{\chi}_{2}^{0} \rightarrow \bar{q} q \ell^{+} \tilde{\ell}^{-} \rightarrow \bar{q} q \ell^{+} \ell^{-} \tilde{\chi}_{1}^{0} .
\end{aligned}
$$

The importance of this is due to the presence of leptons, which allows easier extraction from the background. However, when confronted with leptonic or semi-leptonic R-violating neutralino decay, such leptons will constitute a background for our signals.

We shall, in turn, allow the LSP to decay via $L L \bar{E}, L Q \bar{D}$ and $\bar{U} \bar{D} \bar{D}$ couplings, as depicted in figure 1.

Our analysis has been performed for some of the SPS points [43]. These points have been identified so as to satisfy the WMAP constraint on dark matter [44-46]. While we do not require stable dark matter, these benchmark points are, nevertheless, convenient reference points in the multidimensional parameter space and allow for direct comparisons between the MSSM results in the literature and the expectations in the presence of Rviolating operators. Several of the results we obtain are similar for all these points and therefore, for many of the distributions to be discussed, we focus on the SPS1a case. However, for those predictions that are more sensitive to the SUSY parameter space, we present more global results, elaborating on the differences to be expected in each case.

In the above cascade decay, the actual strength of the R-violating coupling does not lead to qualitative differences in the predictions, as long as: 
(i) it is strong enough for the neutralino decays to be inside the detector $\left(\geq 10^{-6}\right.$ for $100 \mathrm{GeV}$ sparticles and scaled accordingly for higher masses [47], in the case that we do not have any additional effects from phase space suppressions);

(ii) it is not sufficiently large for sparticles to decay directly via the R-violating operator instead of reaching the end of the chain and then decaying via neutralinos.

For couplings closer to $10^{-6}$ there might be displaced vertices that can improve the extraction of the signal $[48,49]$. This, however, has not been studied in this work.

The numerical value of the upper limit of couplings does depend on the SUSY parameter space and the flavour of the R-violating operator under consideration. In section 2.1, we show that the cascade decay actually dominates for a large part of parameter-space as long as the R-violating coupling is kept within experimental bounds. Especially when more than one coupling is large, the strong bounds imposed on products of couplings from flavour violating processes [50-53] will assure cascade dominance.

To analyse the above scenario, we simulate proton-proton collisions at a center of mass energy of $14 \mathrm{TeV}$ using the PYTHIA 8.1 [54] Monte Carlo event generator. In order to correctly describe the kinematical distributions, we have modified PYTHIA to use the energy distributions of appendix A for the neutralino decay. For the SUSY input we use the SUSY Les Houches Accord (SLHA) [55] spectra from SoftSUSY [56], together with SLHA decay tables calculated in PYTHIA 6.4 [57].

The jet analysis is done using FastJet $2.4[58]$ with a $k t$-algorithm $[59,60]$ where the parameter $R_{\text {jet }}$, denoting the upper limit on $\Delta R \equiv \sqrt{(\Delta \eta)^{2}+(\Delta \phi)^{2}}$ for a merge to be allowed, is set to 0.4 unless stated otherwise. Included in the analysis are all detectable particles, i.e. all particles except neutrinos (and stable neutralinos and gravitinos if applicable).

For analyses including taus and b-quarks, the definition of tau (b-) jets is that at least $60 \%$ of the $p_{T}$ of the jet should come from decay products of the tau (b-quark). This fraction is known in the Monte Carlo but for a more realistic study one would need to resort to proper tau and b tagging. For the rest of the paper we shall denote the tau-jets, $\tau_{\text {jet }}$ and the b-jets, $b_{\text {jet }}$, except in figures where we use $\tau$ and $b$ in order to save space.

A lepton is considered isolated if there is no jet with $p_{T}>10 \mathrm{GeV}$ within a cone of $\Delta R<0.4$ and the total additional energy within $\Delta R<0.2$ is less than $10 \mathrm{GeV}$. Note that leptons are included in the jet clustering, but jets that, after the clustering, contain essentially nothing but a lepton, are not included in the further calculations involving jets.

We also impose acceptance cuts, requiring $p_{T}>5 \mathrm{GeV}$ for leptons and $p_{T}>10 \mathrm{GeV}$ for $\tau_{\text {jet }}$ 's and $b_{\text {jet }}$ 's. Where light quark jets are used, they are required to have $p_{T}>20 \mathrm{GeV}$. The reason for the harder cut on light jets as compared to $\tau_{\text {jet }}$ 's and $b_{\text {jet }}$ 's, is to remove some background jets from beam remnants, bremsstrahlung etc.

All data presented is based on simulations of one million events for each operator/SPS point combination. The backgrounds, $t \bar{t}, W, Z$ and QCD (with total $p_{T}>300 \mathrm{GeV}$ ) are studied simulating 10 million events for each in order to account for their larger crosssections. 

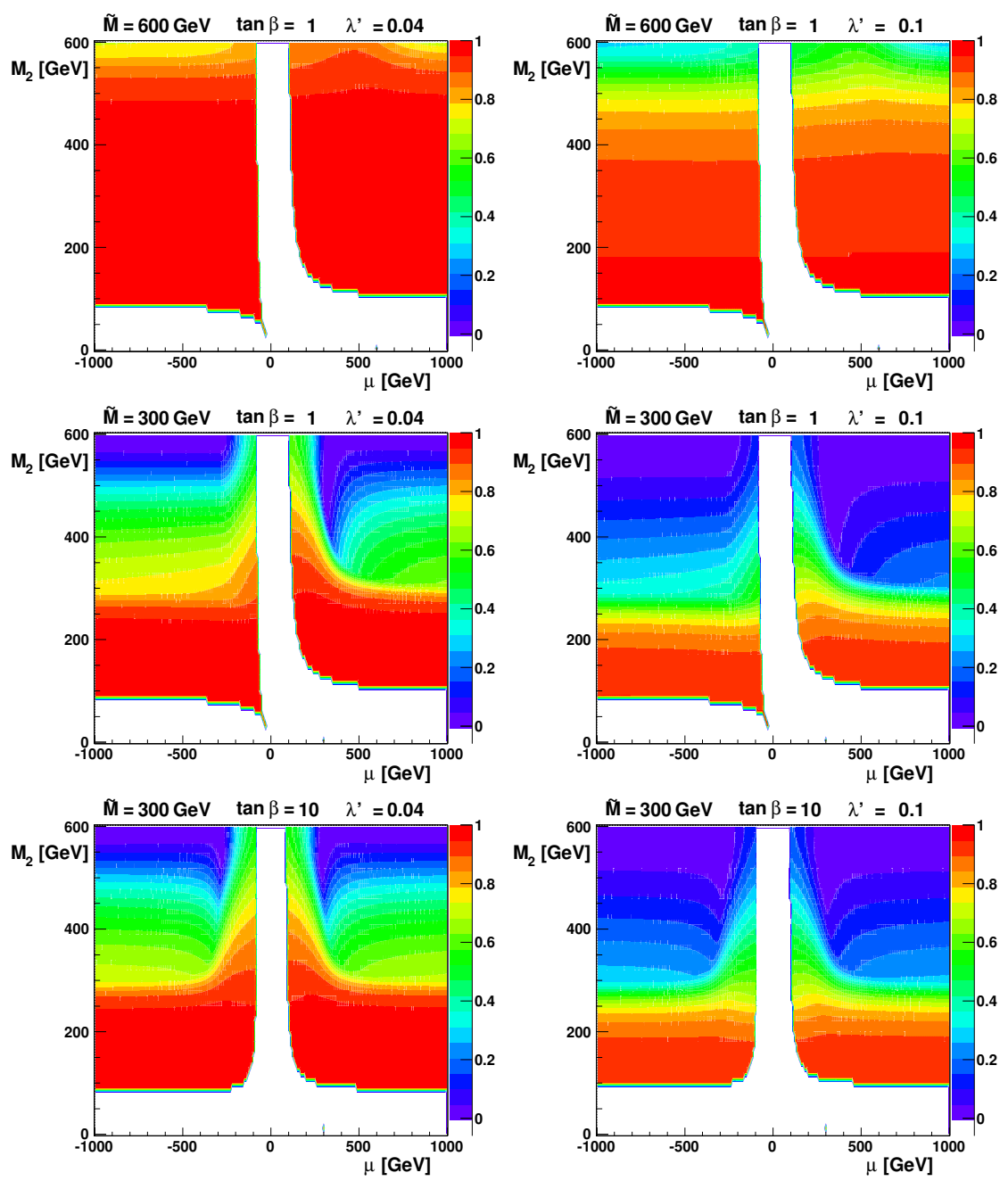

Figure 2. Dominance of MSSM decay of squark vs its R-parity-violating decay, shown for two squark masses, and two values each of $\tan \beta$ and $\lambda^{\prime}$.

\subsection{Cascade dominance}

In order for the cascade to proceed to the end, giving an (unstable) LSP, the competing Rviolating decay of the squark cannot be too strong. The decay of the squark to a quark and a neutralino (or chargino) proceeds via a combination of gauge and Yukawa couplings, in competition with an R-violating decay to a quark and a lepton with a strength proportional to some $\lambda^{\prime}$ squared. ${ }^{5}$ The relative rates of these channels will depend on the squark mass, as well as on the neutralino and chargino spectrum, and the coupling $\lambda^{\prime}$. In order to illustrate this competition, we determine neutralino and chargino spectra in terms of $M_{2}$, $\mu$ and $\tan \beta$ (holding $M_{1}=(5 / 3) \tan ^{2} \theta_{W} M_{2}$ ) and plot, in figure 2 , the ratio

$$
\xi \equiv \frac{\sum \Gamma\left(\tilde{q} \rightarrow q \tilde{\chi}_{i}^{0}\right)+\sum \Gamma\left(\tilde{q} \rightarrow q^{\prime} \tilde{\chi}_{i}^{ \pm}\right)}{\Gamma(\tilde{q} \rightarrow \text { all })} .
$$

\footnotetext{
${ }^{5}$ Analogous statements hold for decays through $\lambda^{\prime \prime}$, and in case of slepton/sneutrino decays for $\lambda$ couplings.
} 
Where this ratio is high (represented in red and yellow), the decay of the squark will proceed via a cascade to the lightest neutralino, which then will decay via the R-parity-violating interaction. Note that we do not need any assumption here about where in the decay chain the studied squark is produced and hence a high $\xi$ for all sparticles present in the coupling, will ensure that the cascade continues down to the lightest neutralino.

The white regions in these panels are excluded by the lightest chargino mass being in conflict with the LEP bound, $M_{\tilde{\chi}_{1}^{ \pm}} \geq 94 \mathrm{GeV}$ [61].

For some representative combinations of the squark mass $\tilde{M}, \tan \beta$ and $\lambda^{\prime}$, we see in figure 2 that even for rather substantial R-violating couplings, the cascade decay dominates for large regions of parameter space. One should also remember that typical decay chains may involve particles other than those present in the dominant R-violating coupling and thus have no open channels for direct R-violating decay. Since these cascades will always proceed to the lightest neutralino, the probability of interrupting the cascade with a direct decay, is further reduced compared to the above discussion.

\section{$3 \quad L L \bar{E}$ couplings}

We shall first consider the couplings $L_{i} L_{j} \bar{E}_{k}$, which, typically, lead to decays of the LSP to two charged leptons and a neutrino, for example

$$
\tilde{\chi}_{1}^{0} \underset{L_{1} L_{2} \bar{E}_{1}}{\longrightarrow} e^{+} \mu^{-} \nu_{e}, \quad e^{-} \mu^{+} \bar{\nu}_{e}, \quad e^{+} e^{-} \nu_{\mu}, \quad e^{+} e^{-} \bar{\nu}_{\mu} .
$$

While isolated leptons in the final state would also arise in R-conserving scenarios from decays higher up in the cascade chain, we will see that differences in kinematic distributions allow us to statistically disentangle the composition of the final state.

We assume that only one $L L \bar{E}$ operator is non-vanishing and set the corresponding coupling to be

$$
\lambda_{i j k}=10^{-4} .
$$

It should be noted that, even if two (or more) couplings of such a size are introduced, the constraints from FCNC [50-53] are satisfied almost trivially. There do exist a few combinations for which even such sizes can be of concern, but these we take care of specifically. Note also that many such constraints actually apply in combinations like $\sum_{i} \lambda_{i j k} \lambda_{i \ell n}^{*} / m_{i}^{2}$, and given that the couplings $\lambda$ can assume either sign (and, in principle, any phase), larger couplings could, in principle, be accommodated if one were to admit cancellations. Collider signals, on the other hand, would be insensitive to such cancellations. We, however, desist from considering such possibilities. Identical observations would hold for $\lambda^{\prime}$ couplings as well, while, for $\lambda^{\prime \prime}$ couplings, the bounds are, in general, even weaker. However, we will return to the issue of a comparative study of the magnitude of the operators, if several of them are large - a main aim of the paper - in a subsequent section.

For the analysis of the $L L \bar{E}$ operators, the following selection cuts have been used [62, $63]$ :

- at least three isolated leptons with $p_{\mathrm{T}}>20 \mathrm{GeV}$; 


\begin{tabular}{|c|c|c|c|c|c|c|}
\hline$i j k$ & $e e$ & $\mu \mu$ & $\tau \tau$ & $e \mu$ & $e \tau$ & $\mu \tau$ \\
\hline 121 & $\checkmark$ & & & $\checkmark$ & & \\
\hline 122 & & $\checkmark$ & & $\checkmark$ & & \\
\hline 123 & & & & & $\checkmark$ & $\checkmark$ \\
\hline 131 & $\checkmark$ & & & & $\checkmark$ & \\
\hline 132 & & & & $\checkmark$ & & $\checkmark$ \\
\hline 133 & & & $\checkmark$ & & $\checkmark$ & \\
\hline 231 & & & & $\checkmark$ & $\checkmark$ & \\
\hline 232 & & $\checkmark$ & & & & $\checkmark$ \\
\hline 233 & & & $\checkmark$ & & & $\checkmark$ \\
\hline
\end{tabular}

Table 1. Possible final states for $L L \bar{E}$ couplings.

- the leading lepton has $p_{\mathrm{T}}>70 \mathrm{GeV}$;

- $p_{\mathrm{T}}^{\text {miss }}>100 \mathrm{GeV}$.

These cuts are sufficient to reduce $t \bar{t}$ events by a factor $\approx 3 \cdot 10^{-6}$ and $W$ and $Z^{0}$ events (including all production of weak bosons) by a factor $\approx 6 \cdot 10^{-5}$. The RPV events, on the other hand, are reduced to approximately $1-40 \%$ of the total, with the suppression factor depending on the point in the SUSY parameter space as well as the nature of the RPV interaction. For operators with a lot of tau flavour, the reduction is significantly stronger than for operators involving only light flavours.

This means that in the case of a SUSY parameter point with low cross section and low lepton production in combination with a tau-rich $L L \bar{E}$ operator, we might get some $W$ and $Z^{0}$ events in the sample, but detailed simulations show that this contamination in the studied distributions is not a significant problem.

Overall, in the presence of R-violation via $L L \bar{E}$ operators, we can see spectacular signatures (final-state flavours, like-sign leptons, etc.) $;{ }^{4}$ these become more pronounced for the $L_{2} L_{3} \bar{E}_{1}$ and $L_{1} L_{3} \bar{E}_{2}$ couplings, which mix all flavours.

\subsection{Final-state flavour correlations}

Since we cannot detect the neutrinos or their flavours, the characteristics of various decay channels have to be described in terms of the charged leptons in the decay. From each neutralino we get one pair of charged leptons and their identities are correlated with the flavours in the operator.

It is important to remember that every operator gives rise to two distinct channels, a consequence of the fact that one of the charged leptons is associated with the $\bar{E}$ superfield and the other with the $L$ superfield. Since $\mathrm{SU}(2)$ invariance requires the $L$-type fields to have different flavours, this leads to exactly two different channels, modulo charge conjugation. The operator $L_{1} L_{2} \bar{E}_{1}$, for example, will give both $e^{+} e^{-}$and $e \mu$ pairs with equal frequency. 

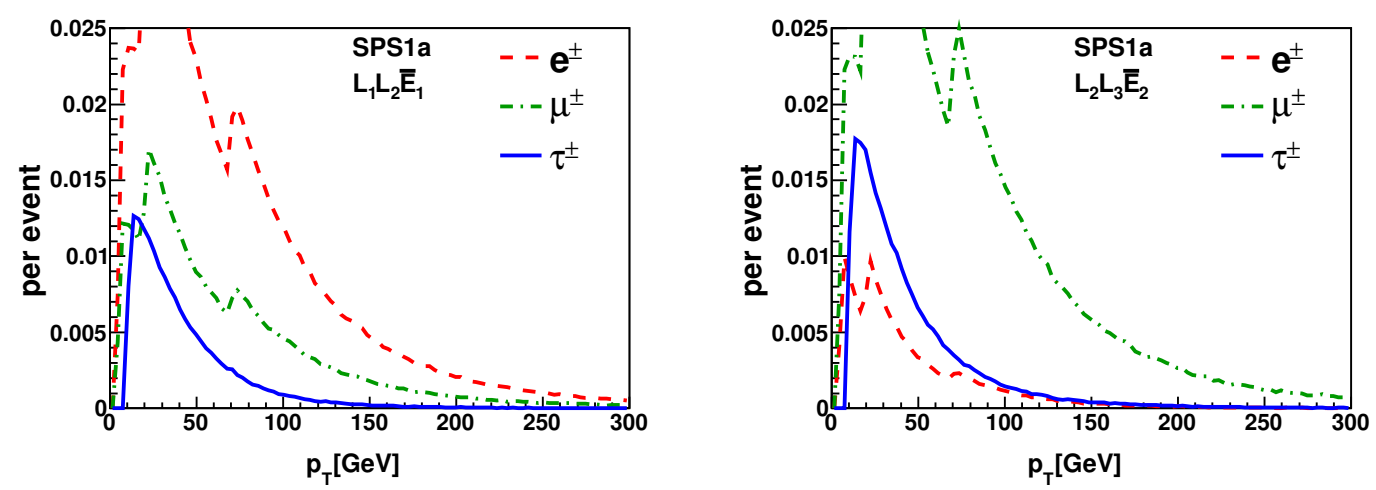

Figure 3. Electron, muon and $\tau_{\text {jet }}$ spectra for $L_{1} L_{2} \bar{E}_{1}$ (left panel) and $L_{2} L_{3} \bar{E}_{2}$ (right panel) at SPS1a. Notice the dips in the spectra at $20 \mathrm{GeV}$; this comes from the fact that a lepton with $p_{T}<20 \mathrm{GeV}$ can only pass the cuts if there are at least 3 other high $p_{T}$ leptons in the event. One can also see features at $70 \mathrm{GeV}$ due to the cut.

A further complication arises when tau fields are involved, then light-lepton pairs may arise from leptonic tau decays. And since our final states require isolated light leptons, the leptonic tau decays will be favoured over hadronic ones. Similarly, decay channels wherein the superparticles decay directly into light leptons would have a higher detection efficiency compared to those involving intermediate tau states. Thus, the composition of final states measured is not the same as given directly by the branching fractions but depends nontrivially on the supersymmetric spectrum as well as the flavour structure of the coupling.

The open channels for each coupling are given in table 1 .

\section{$3.2 \quad p_{T}$ distributions}

We first look at the $p_{T}$ spectra of the electrons (henceforth, "electrons" will refer collectively to both electrons and positrons) and muons as well as hadronic taus. We shall assume that hadronic taus $\left(\tau_{\text {jet }}\right)$ can be identified (with some efficiency). Note that in the $\tau_{\text {jet }} \mathrm{s}$ the neutrino is not included.

The electron, muon and $\tau_{\text {jet }}$ spectra for $L_{1} L_{2} \bar{E}_{1}$ and $L_{2} L_{3} \bar{E}_{2}$ at SPS1a are shown in figure 3. The two panels look the same apart from different relative normalizations of individual curves. These different normalizations can give insight into the flavour composition of the operator. However, since we do not know how much the R-conserving decay chain contributes and also since the overall number of leptons is more or less dictated by the cuts, the most interesting information here might be the difference in normalization between the electron and the muon spectra. Since the R-conserving chain is not expected to give any difference here, it might give some hint on the dominant flavour in the coupling.

\subsection{Invariant mass distributions}

One of the best tools for studying leptonic decays of the neutralino are invariant mass distributions. The charged leptons from the decay will give rise to invariant mass distributions as calculated in appendix B. Since the flavours of the involved leptons are determined by 
the flavours in the coupling, the channels in which these distributions are to be expected, can be deduced from table 1 .

Let us illustrate how this works: $L_{2} L_{3} \bar{E}_{1}$ gives rise to $e^{ \pm} \mu^{\mp}$ and $e^{ \pm} \tau^{\mp}$ final states (see table 1). Therefore, we expect a prompt contribution of the type (B.2) in the $e \mu$ invariant mass distribution as well as contributions of the type (B.11) (from leptonic tau decays) in the $e \mu$ as well as $e e$ distributions. In addition, the $e^{ \pm} \tau^{\mp}$ final state should give a contribution of type (B.12) to the $e \tau_{\text {jet }}$ distribution.

Since our goal is to determine the flavours of the coupling, we want to study all dilepton distributions available, as well as distributions including $\tau_{\text {jet }}$ 's. We then want to use the presence of the various signals together with table 1 to determine the operator flavours.

Since we cannot tell which leptons come from the same vertex, we will have a problem with unrelated lepton pairs, i.e., pairs of leptons from different parts of the event. This combinatorial background will, in some cases, make it difficult to see the signal. However, since these pairs are not related, they will equally often have the same charge as the opposite, and since we do not expect any signals in the same-sign distributions, subtracting those distributions turns out to be a very effective way of removing these backgrounds.

The effect of the same-sign subtraction can be seen in figure 4 where we show the dilepton distributions for $L_{2} L_{3} \bar{E}_{1}$ at SPS1a both without and with same-sign (SS) subtraction. From this we can see that the same-sign subtraction is especially important in order to extract the smaller signals where leptonically decaying taus are involved. Also for the distributions involving $\tau_{\text {jet }}$ 's it is crucial to apply same-sign subtraction in order to see the signals.

\subsection{Backgrounds and uncertainties}

The interpretation of any detected excess as a signal is possible only if we understand well the (backgrounds from) R-conserving decay chains and also the effect of the cuts on the shapes of the distributions. The latter issue is expected to affect the accuracy of the fit of the theoretical distributions to the data, which makes, e.g., a reconstruction of the neutralino mass more challenging. The first issue, on the other hand, depends on the SUSY model and is especially important for the distributions including $\tau_{\text {jet }}$ 's where the contributions from the R-conserving chains look very similar to the contributions from the neutralino decay. This can be seen in figure 4 where both the $\mu \tau_{\text {jet }}$ and the $\tau_{\text {jet }} \tau_{\text {jet }}$ distributions show unexpected features, closely resembling the expected signals. On the other hand, the corresponding triangular distributions in the $e e$ and $\mu \mu$ distributions are more distinct and can therefore be removed from the analysis (practically invisible in figure 4).

There are, of course, backgrounds from standard-model processes too, but these are well handled by the event selection criteria. Other experimental issues include photon conversion as well as photon and lepton misidentification. The necessity of same-sign subtraction also implies a sensitivity to the ability to correctly measure the sign of the leptons, especially the corresponding charge of $\tau_{\text {jet }}$ 's.

For some choices of SUSY parameters, a real $Z^{0}$ may be produced in the decay chain and this would show up in the same-flavour (opposite-sign) dilepton invariant mass distributions. However, since this only gives a peak at the $Z^{0}$ mass, this effect can be easily accounted for. 

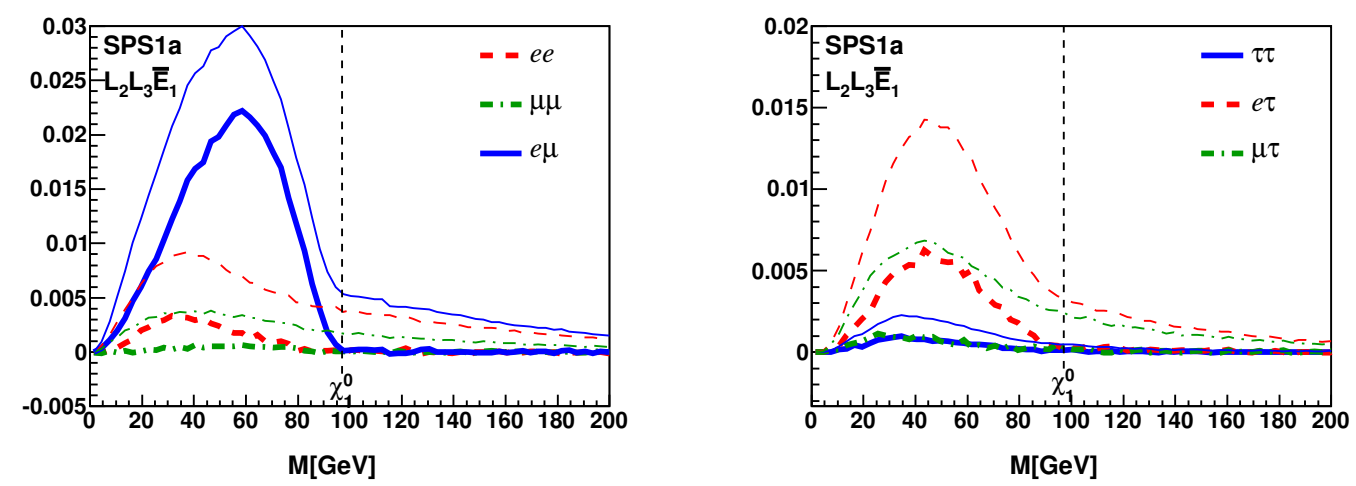

Figure 4. Di-lepton invariant masses for $L_{2} L_{3} \bar{E}_{1}$ at SPS1a, where the neutralino mass is $97.0 \mathrm{GeV}$. Thin curves: without same-sign subtraction. Heavy curves: $M\left(\ell \ell^{\prime}\right)$ distributions after same-sign subtraction. Here, " $\tau$ " refers to a hadronic tau.

\subsection{Comparison of Monte Carlo results with theory}

We now compare Monte Carlo simulations with the theoretical distributions derived in appendix B. In what follows, the neutralino mass and the normalizations of the spectra are fitted to the Monte Carlo distributions, thereby allowing for a determination of the neutralino mass.

As can be seen in e.g. figure 20 in appendix B, most of the expected distributions look very similar, the main differences being different kinematical cutoffs. This means we have to be careful with the fitting procedure to avoid too large degeneracies in the fitted parameters; e.g. a distribution of type (B.2) may look very similar to a distribution of type (B.11) with a larger neutralino mass.

To avoid problems with this, each distribution has only been fitted using the distributions that are relevant due to the assumed operator flavours. For the relevant distributions we also include a triangular distribution where the cutoff is assumed known (only for SUSY models where such a contribution from the chain exists), and a peak at the $Z^{0}$ mass. The parameters to be fitted are then the neutralino mass as well as the normalizations of the various components.

In a realistic scenario where the operator is not known but to be determined, the relevant distributions can be determined by a comparison with Monte Carlo data. In cases where this is difficult one can exploit the expected correlations between the distributions; if one distribution has a clear cutoff, the others cannot have neutralino masses significantly lower than that cutoff. Note that at least one of the distributions will always have a cutoff close to the neutralino mass.

Assuming $L_{2} L_{3} \bar{E}_{1}$ to be the dominant R-violating term, various dilepton mass distributions corresponding to the benchmark points SPS1a, SPS1b and SPS6 are shown in figure 5. The left panels show the pure dilepton invariant mass distributions while the right panels show the distributions involving $\tau_{\text {jet }}$ 's. As one can see, the fits are in all cases quite good, but there are some deviations at the lower end, especially in the $\tau_{\text {jet }}$ distributions. 


\begin{tabular}{|l|r|r|r|r|r|r|r|}
\hline \multicolumn{1}{|c|}{ model } & \multicolumn{1}{c|}{$M_{\chi_{1}^{0}}$} & \multicolumn{1}{c|}{$e e$} & \multicolumn{1}{c|}{$\mu \mu$} & \multicolumn{1}{c|}{$e \mu$} & \multicolumn{1}{c|}{$e \tau$} & \multicolumn{1}{c|}{$\mu \tau$} & \multicolumn{1}{c|}{$\tau \tau$} \\
\hline$L_{2} L_{3} \bar{E}_{1}, \mathrm{SPS} 1 \mathrm{a}$ & 97.0 & $116 \pm 0.3$ & - & $96.8 \pm 0.1$ & $106 \pm 0.5$ & - & - \\
$L_{2} L_{3} \bar{E}_{1}, \mathrm{SPS} 1 \mathrm{~b}$ & 162.2 & $167 \pm 0.5$ & - & $171 \pm 0.1$ & $171 \pm 0.4$ & - & - \\
$L_{2} L_{3} \bar{E}_{1}, \mathrm{SPS} 6$ & 189.8 & $195 \pm 1$ & - & $187 \pm 0.1$ & $200 \pm 0.5$ & - & - \\
$L_{1} L_{3} \bar{E}_{3}, \mathrm{SPS} 6$ & 189.8 & $205 \pm 0.2$ & $295 \pm 6$ & $195 \pm 1$ & $198 \pm 0.8$ & $227 \pm 2$ & $224 \pm 3$ \\
$L_{1} L_{3} \bar{E}_{3}, \mathrm{SPS} 1 \mathrm{~b}$ & 162.2 & $172 \pm 2$ & $272 \pm 13$ & $176 \pm 0.4$ & $172 \pm 0.7$ & $204 \pm 3$ & $187 \pm 3$ \\
$L_{1} L_{2} \bar{E}_{3}, \mathrm{SPS} 1 \mathrm{~b}$ & 162.2 & $173 \pm 1$ & $172 \pm 1$ & $172 \pm 0.5$ & $172 \pm 0.4$ & $172 \pm 0.4$ & - \\
\hline
\end{tabular}

Table 2. Fitted neutralino mass (all values are in $\mathrm{GeV}$ ) from the various distributions.

This is due to the cuts imposed and the consequent deterioration of the signal profile. Especially in the case of SPS6 we find that the inclusion of the triangular contribution from the chain into the fit is crucial for a correct fit to the smaller signals.

The situation changes somewhat when the operators give large numbers of taus in the decay. However, as figure 6 shows, the fit is still rather good. Of special interest might be the case of $L_{1} L_{3} \bar{E}_{3}$ at SPS6 where there is no tau from the cascade decay chain and we can therefore see all the distributions expected, including the softer $\mu \tau_{\text {jet }}$ signal where the $\mu$ comes from a decaying tau. When comparing the various couplings at SPS1b it is clear that one cannot tell whether a signal in the $\tau_{\text {jet }} \tau_{\text {jet }}$ distribution is from the neutralino decay or the decay chain, the expected distributions are much too similar.

Table 2 shows the neutralino masses estimated from the fits shown in figures 5 and 6 . Included are also statistical errors on the fitted neutralino masses. In addition we expect systematic uncertainties from the theory, especially concerning the inclusion of the event selection. Therefore, the uncertainties given in table 2 should not be taken too seriously but rather as lower bounds on the uncertainty.

It seems that the measurement of the neutralino mass from the $e \tau_{\text {jet }}$ mass always gives an overestimate. The same is true for the di-lepton mass distributions when one of the leptons comes from a decaying tau, although, in the latter case, the overestimate is less severe. In the relevant cases, the distribution when both leptons come from decaying taus are included but the impact on the resulting fit is not very large; we see from the $\mu \mu$ distribution for the $L_{1} L_{3} \bar{E}_{3}$ operator, where both muons have to come from decaying taus, that the signal is too small to get a good fit and the resulting neutralino mass estimate is completely useless. This is reflected in the very large statistical uncertainty. In general the possible improvement by including this last distribution is smaller than the uncertainties from e.g. the effects of the event selection.

When interpreting these neutralino mass estimates, one has to keep in mind the large uncertainty associated with the effects of the event selection on the distribution. In the theoretical curves, these effects are included through the lower limit on the mass ratio entering in eq. (B.11). The value of this limit has great impact on the resulting distribution and therefore on the estimated neutralino mass. It is therefore clear that a better understanding of the effect of the cuts is very important in order to get the best possible neutralino mass estimate. 

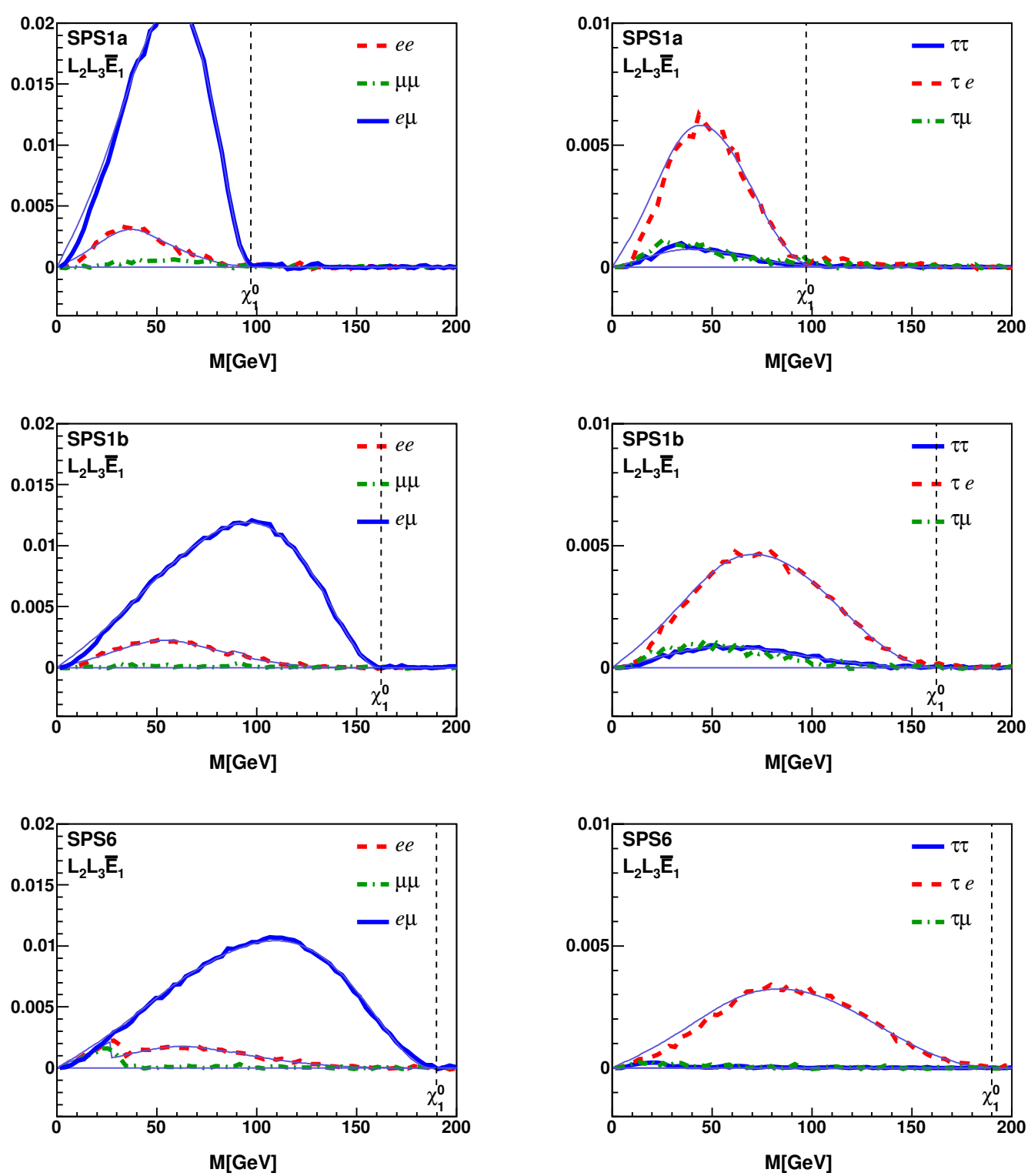

Figure 5. Comparison between fitted theoretical distributions (thin curves in violet) and Monte Carlo results (heavy curves). The results for $L_{2} L_{3} \bar{E}_{1}$ are shown for three different SPS points.

In order to improve these fits one could make use of various expected correlations; e.g. as mentioned before, the neutralino mass of course has to be the same for all distributions and one could use the most suitable distribution, presumably the one with a cutoff at the neutralino mass, to constrain the fits to the smaller distributions with cutoffs significantly below the neutralino mass. Ideally one would want to use the fitted parameters to determine the decay channels for the neutralino, but due to the aforementioned degeneracy problems it is hard to achieve the required accuracy in the parameters, this could be improved if one uses the fact that e.g. an $e \tau$ decay channel for the neutralino gives correlated 

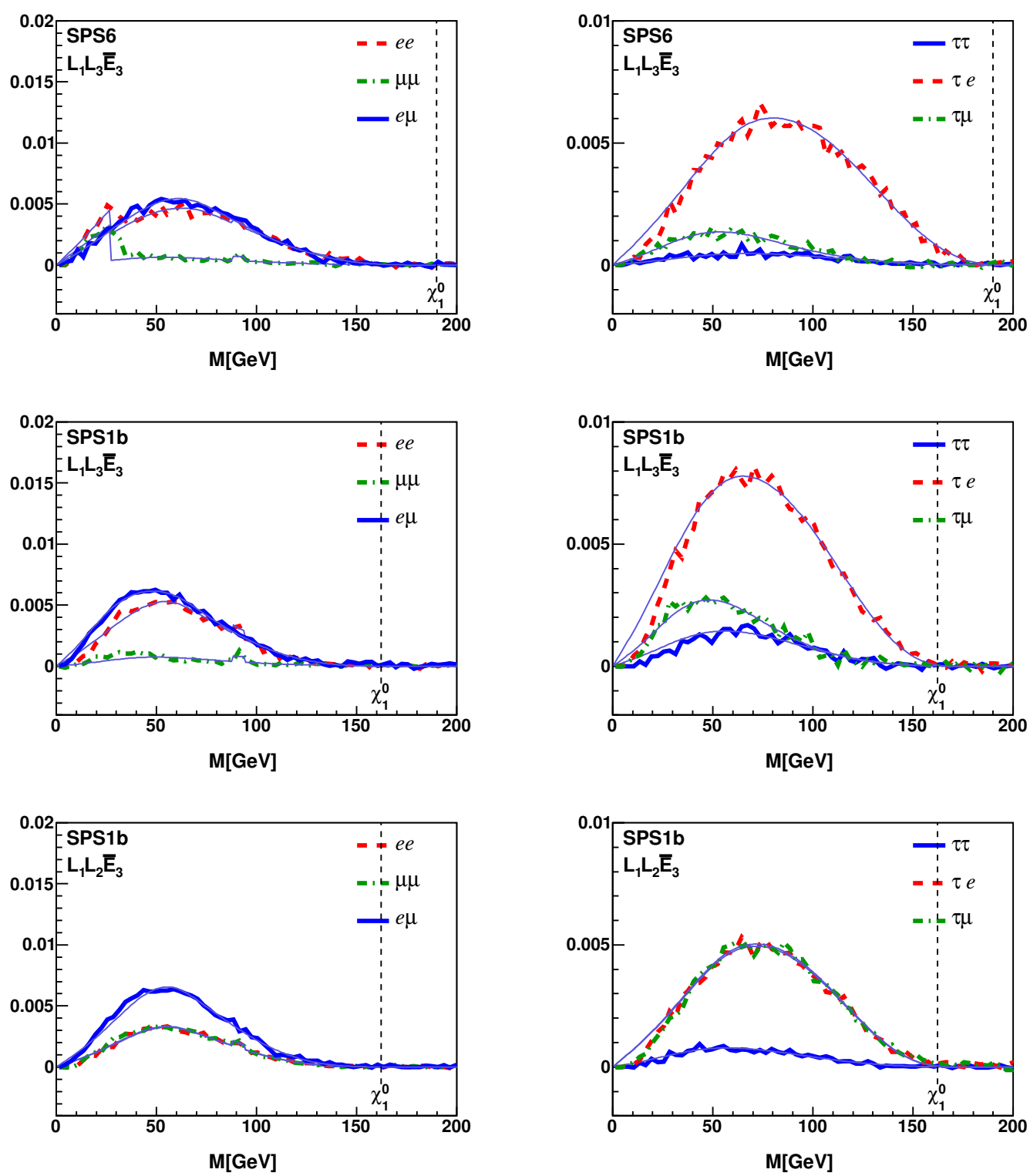

Figure 6. The same as figure 5 for some tau-rich operators at SPS1b and SPS6.

signals in both the $e \tau_{\text {jet }}$, $e e$ and $e \mu$ distributions, where the relative sizes of the signals are basically given by the branching fractions of the tau (together with tau tagging efficiency and detector acceptances).

\subsection{Some counting observables}

We now consider observables that can bring additional insight into the operator structure. One obvious candidate is the determination of the branching fractions of the neutralino into various channels. This can be achieved by studying the leptonic structure of the final states. 
To get a clean sample to work with, we look at the subset of all events where we have exactly four leptons of which two are positively charged and two are negatively charged. This restriction increases the probability that the leptons have all come from decaying neutralinos.

Let $P_{2 e^{-} 2 e^{+}}$denote the fraction of these events that have exactly two electrons and two positrons:

$$
P_{2 e^{-} 2 e^{+}}=\frac{N\left[\left(2 e^{-}\right) \&\left(2 e^{+}\right)\right]}{N_{4 \ell}}
$$

(and similarly for $P_{2 \mu^{-}} 2 \mu^{+}$), with

$$
N_{4 \ell}=N[(4 e)]+N[(3 e) \&(1 \mu)]+N[(2 e) \&(2 \mu)]+N[(1 e) \&(3 \mu)]+N[(4 \mu)],
$$

subject to the constraint that the charges should sum to zero. Further, let $P_{2 e 2 \mu}$ denote the fraction that has two same-sign electrons and two same-sign muons, i.e., $2 e^{-} 2 \mu^{+}$or $2 e^{+} 2 \mu^{-}$:

$$
P_{2 e 2 \mu}=\frac{N\left[\left(2 e^{-}\right) \&\left(2 \mu^{+}\right)\right]+N\left[\left(2 e^{+}\right) \&\left(2 \mu^{-}\right)\right]}{N_{4 \ell}} .
$$

In the event that all four leptons come from decaying neutralinos, $P_{e^{-}} e^{+}$is the probability that a neutralino decays to an electron-positron pair. Since under these assumptions, the only possibility to achieve $2 e^{-} 2 e^{+}$in one event is to have two neutralinos that both decay to electron-positron pairs, we get

$$
P_{2 e^{-} 2 e^{+}}=P_{e^{-} e^{+}}^{2}
$$

Note that $P_{e^{-}} e^{+}$is a probability ${ }^{6}$ referring to one neutralino, whereas $P_{2 e^{-}} 2 e^{+}$refers to the whole event.

Similarly, $P_{2 \mu^{-} 2 \mu^{+}}=P_{\mu^{-} \mu^{+}}^{2}$ and $P_{2 e 2 \mu}=\frac{1}{2} P_{e \mu}^{2}$ where $P_{e \mu}=P_{e^{-} \mu^{+}}+P_{e^{+} \mu^{-}}$and $P_{\mu^{-} \mu^{+}}, P_{e^{-} \mu^{+}}$and $P_{e^{+} \mu^{-}}$are defined in accordance with $P_{e^{-} e^{+}}$. The factor $\frac{1}{2}$ in the second of these equations comes from the requirement that the same-flavour pairs also have the same charge. From this we can calculate the values $P_{e^{-} e^{+}}, P_{\mu^{-} \mu^{+}}$and $P_{e \mu}$.

A problem with the above calculation is that it does not tell us anything about channels including taus. Since we require exactly four leptons, events with taus are only included when the taus decay leptonically. This also means that decay channels with light leptons are favoured by the cuts over those with taus and therefore care has to be taken when interpreting the numbers. However, keeping this in mind, the probabilities $P_{e^{-} e^{+}}, P_{\mu^{-} \mu^{+}}$ and $P_{e \mu}$ do tell us which decay channels are important.

In order to get some information about decay channels involving taus, we need to also study events with three rather than four leptons. Let us study events with one pair of same-sign, same-flavour leptons $\left(\ell^{ \pm} \ell^{ \pm}\right)$and one extra lepton of opposite charge and different flavour $\left(\ell^{\prime} \mp\right)$. Such events must all contain one different-flavour, opposite-sign lepton pair $\left(\ell^{ \pm} \ell^{\prime} \mp\right)$ and one extra lepton $\left(\ell^{ \pm}\right)$, the extra lepton stemming from a neutralino

\footnotetext{
${ }^{6}$ This is the probability that a randomly picked neutralino in an event with exactly four leptons, is decaying through a certain channel. In other words; it is a branching fraction, but depends strongly on the cuts used to calculate it and it should not be confused with a "true" branching fraction.
} 
that decayed either to a $\tau$ plus a lepton where the $\tau$ decayed hadronically, or to a lepton pair where one of the leptons was lost to the detector.

Since counting observables depend not only on production rate and detector efficiencies, but also on a priori unknown factors such as selection efficiencies, it is useful to study, instead, ratios such as

$$
\frac{P_{2 e \mu}}{P_{2 \mu e}} \equiv \frac{N_{2 e^{-} \mu^{+}}+N_{2 e^{+} \mu^{-}}}{N_{2 \mu^{-} e^{+}}+N_{2 \mu^{+} e^{-}}}=\frac{N_{2 e^{-} \mu^{+}}}{N_{2 \mu^{-} e^{+}}}
$$

where the numbers $N$ refer to the number of events with the specified number of leptons. The last equality above is based on the assumption that both charges are produced with the same probability and detected with the same efficiency.

Let us now consider a particular case wherein we start with four leptons $(e, \mu)$ stemming directly from the decays of two neutralinos with one of the four (randomly picked) getting lost (absorbed in a jet, too little $p_{T}$, too large a rapidity etc.). The flavour distribution of the four-lepton state is then given by the probabilities $P_{\mu^{-} \mu^{+}}, P_{e \mu}$ and $P_{e^{-}} e^{+}$. Assuming that the efficiencies for muons are the same as for electrons, ${ }^{7}$ we have

$$
\frac{P_{2 e \mu}}{P_{2 \mu e}}=\frac{P_{e \mu}\left(2 P_{e^{-} e^{+}}+P_{e \mu}\right)}{P_{e \mu}\left(2 P_{\mu^{-} \mu^{+}}+P_{e \mu}\right)}=\frac{2 P_{e^{-} e^{+}}+P_{e \mu}}{2 P_{\mu^{-} \mu^{+}}+P_{e \mu}},
$$

where the factor 2 is a combinatorial factor arising from the fact that the individual leptons could have arisen from the two different neutralinos.

Since the prediction of eq. (3.8) does not take into account the possibility of the fourth missing particle being a hadronically decaying tau, it will, occasionally, give an inaccurate answer. This apparent lacuna could, however, be used to our advantage: a comparison of the prediction of (3.8) with a direct measurement of the ratio (3.7) would give us a quantitative measure of the tau decay channel. If the decay channel $\chi_{1}^{0} \rightarrow e+\tau+\nu$ is a significant one, the measured fraction will be much larger than the prediction (3.8), while if the channel $\chi_{1}^{0} \rightarrow \mu+\tau+\nu$ is present, eq. (3.8) will give an overestimate.

Independent measures of the branching fractions to various final states are given by the integrals over invariant mass, namely

$$
\int \mathcal{N}_{l l^{\prime}} \equiv \int_{0}^{\infty} \frac{d \mathcal{N}_{l l^{\prime}}\left(M_{l l^{\prime}}\right)}{d M_{l l^{\prime}}} d M_{l l^{\prime}}
$$

where $l, l^{\prime} \in\{e, \mu\}$ and the distributions are evaluated after same-sign subtraction. Whereas the $P$ 's above refer to the four-lepton events where the charges sum to zero, these integrals refer to all events that pass the cuts.

Both the integrals over the invariant mass distributions and the probabilities $P_{e^{-} e^{+}}, P_{\mu^{-} \mu^{+}}$and $P_{e \mu}$ must be interpreted with the used cuts in mind; in both cases leptons are favoured over taus. The resulting branching fractions include leptonically decaying taus in addition to prompt leptons but the former contribution is strongly dependent on the cuts and therefore hard to estimate analytically. Due to the requirement of exactly four

\footnotetext{
${ }^{7}$ While this is not exactly true, the differences are marginal, and, at the level of sophistication of this study, can be ignored.
} 


\begin{tabular}{|l|r|r|r|r|r|r|r|r|}
\hline model & $P_{e^{-} e^{+}}$ & $P_{e \mu}$ & $P_{\mu^{-} \mu^{+}}$ & $(3.7)$ & $(3.8)$ & $\int \mathcal{N}_{e e}$ & $\int \mathcal{N}_{e \mu}$ & $\int \mathcal{N}_{\mu \mu}$ \\
\hline$L_{1} L_{2} \bar{E}_{1}$, SPS1a & 0.48 & 0.51 & 0.02 & 2.5 & 2.69 & 0.48 & 0.51 & 0.01 \\
$L_{1} L_{2} \bar{E}_{2}$, SPS1a & 0.02 & 0.49 & 0.50 & 0.37 & 0.35 & 0.01 & 0.49 & 0.50 \\
$L_{1} L_{2} \bar{E}_{3}$, SPS1a & 0.26 & 0.48 & 0.27 & 0.93 & 0.98 & 0.27 & 0.47 & 0.26 \\
$L_{1} L_{3} \bar{E}_{1}$, SPS1a & 0.81 & 0.16 & 0.01 & 19.3 & 10.1 & 0.86 & 0.12 & 0.03 \\
$L_{1} L_{3} \bar{E}_{2}$, SPS1a & 0.02 & 0.81 & 0.17 & 0.26 & 0.75 & 0.02 & 0.87 & 0.11 \\
$L_{1} L_{3} \bar{E}_{3}$, SPS1a & 0.45 & 0.45 & 0.09 & 3.77 & 2.15 & 0.43 & 0.44 & 0.13 \\
$L_{2} L_{3} \bar{E}_{1}$, SPS1a & 0.17 & 0.81 & 0.03 & 3.26 & 1.33 & 0.11 & 0.87 & 0.02 \\
$L_{2} L_{3} \bar{E}_{2}$, SPS1a & 0.01 & 0.16 & 0.82 & 0.05 & 0.10 & 0.02 & 0.11 & 0.86 \\
$L_{2} L_{3} \bar{E}_{3}$, SPS1a & 0.09 & 0.45 & 0.45 & 0.27 & 0.47 & 0.12 & 0.44 & 0.44 \\
\hline
\end{tabular}

Table 3. The three first columns give calculated values of $P_{e^{-}} e^{+}, P_{\mu^{-} \mu^{+}}$and $P_{e \mu}$ for all the different operators at SPS1a. We also give the Monte Carlo value of the fraction (3.7) as well as the prediction from eq. (3.8). Finally, we tabulate the integrals of the invariant mass distributions, normalized so that the sum is one. This should provide an independent estimate of the branching fractions of the first three columns.

\begin{tabular}{|l|r|r|r|r|r|r|r|r|}
\hline model & $P_{e^{-} e^{+}}$ & $P_{e \mu}$ & $P_{\mu^{-} \mu^{+}}$ & $(3.7)$ & $(3.8)$ & $\int \mathcal{N}_{e e}$ & $\int \mathcal{N}_{e \mu}$ & $\int \mathcal{N}_{\mu \mu}$ \\
\hline$L_{2} L_{3} \bar{E}_{1}$, SPS1a & 0.17 & 0.81 & 0.03 & 3.26 & 1.33 & 0.11 & 0.87 & 0.02 \\
$L_{2} L_{3} \bar{E}_{1}$, SPS1b & 0.18 & 0.80 & 0.02 & 3.72 & 1.38 & 0.12 & 0.87 & 0.01 \\
$L_{2} L_{3} \bar{E}_{1}$, SPS6 & 0.17 & 0.81 & 0.03 & 3.54 & 1.33 & 0.12 & 0.85 & 0.03 \\
\hline$L_{1} L_{2} \bar{E}_{3}$, SPS1a & 0.26 & 0.48 & 0.27 & 0.93 & 0.98 & 0.27 & 0.47 & 0.26 \\
$L_{1} L_{2} \bar{E}_{3}$, SPS1b & 0.25 & 0.50 & 0.25 & 1.00 & 0.99 & 0.25 & 0.50 & 0.26 \\
\hline$L_{1} L_{3} \bar{E}_{3}$, SPS1a & 0.45 & 0.45 & 0.09 & 3.77 & 2.15 & 0.43 & 0.44 & 0.13 \\
$L_{1} L_{3} \bar{E}_{3}$, SPS1b & 0.43 & 0.49 & 0.08 & 3.37 & 2.08 & 0.41 & 0.50 & 0.08 \\
\hline
\end{tabular}

Table 4. Similar to table 3 for $L_{2} L_{3} \bar{E}_{1}$ at SPS1a, SPS1b and SPS6, as well as $L_{1} L_{2} \bar{E}_{3}$ and $L_{1} L_{3} \bar{E}_{3}$ at SPS1a and SPS1b.

leptons, the probabilities $P_{e^{-} e^{+}}, P_{\mu^{-} \mu^{+}}$and $P_{e \mu}$ essentially include only events where there are no taus in the decay or all taus decay leptonically. The invariant mass distributions, on the other hand, are expected to contain a larger amount of lepton pairs from sources other than neutralino decay. This can, however, be handled by the same-sign subtraction as well as by comparing the distributions with the theoretical expectations. The probabilities $P_{e^{-} e^{+}}, P_{\mu^{-} \mu^{+}}$and $P_{e \mu}$ are more sensitive to contamination by single leptons from other sources and are therefore more dependent on a clean event sample.

All the observables discussed in this section for all $L L \bar{E}$ couplings at SPS1a are shown in table 3. When comparing the probabilities $P_{e^{-} e^{+}}, P_{\mu^{-} \mu^{+}}$and $P_{e \mu}$ and the integrals over invariant mass distributions, we see that they show similar structures, implying that they both are good indications for the relevant decay channels. 
In table 4 , we compare these observables for the same operator $\left(L_{2} L_{3} \bar{E}_{1}\right)$ but at three different parameter points: SPS1a, SPS1b and SPS6. As the values are similar at all three points, we may conclude that they are largely independent of the underlying SUSY model.

However, also shown in table 4 are $L_{1} L_{2} \bar{E}_{3}$ and $L_{1} L_{3} \bar{E}_{3}$ at SPS1a and SPS1b and here some noticeable differences arise. First of all, for $L_{1} L_{2} \bar{E}_{3}$ we naively expect to get 0.25 , 0.5 and 0.25 for the branching fractions to $e^{+} e^{-}, e \mu$ and $\mu^{+} \mu^{-}$respectively, a consequence of the fact that the tau will go as often to a $\mu$ as an $e$. As can be seen in table 4 this is what we get at SPS1b while SPS1a gives slightly larger values for the same-flavour (ee and $\mu \mu$ ) channels. This difference is consistent with contamination by lepton pairs from the cascade decay chain, which is important for SPS1a but not for SPS1b.

Since this kind of contamination will be most important for tau-rich operators where there are fewer leptons from the neutralino decay, we also expect to see it in $L_{1} L_{3} \bar{E}_{3}$, which, in fact, we do. For a $L_{1} L_{3} \bar{E}_{3}$ coupling, we expect the same amount of $e^{+} e^{-}$and $e \mu$ from the $e \tau$ channel and from the $\tau \tau$ channel we expect contributions to $e^{+} e^{-}, e \mu$ and $\mu^{+} \mu^{-}$ in the ratios $1: 2: 1$ (again due to the two possibilities for $e \mu$ ). The result should be that the branching fraction to $e \mu$ is larger than the fraction to $e^{+} e^{-}$by about the same amount as the total branching fraction to $\mu^{+} \mu^{-}$and this is rather close to what we see for SPS1b, but at SPS1a the $e^{+} e^{-}$and $e \mu$ values are of the same size, indicating some contamination in the $e^{+} e^{-}$(and $\mu^{+} \mu^{-}$) channel from the cascade chain.

Four out of the nine $L L \bar{E}$ operators are such that they allow one channel with prompt light leptons and one channel with a light lepton and a tau. These operators are $L_{1} L_{3} \bar{E}_{1}$, $L_{1} L_{3} \bar{E}_{2}, L_{2} L_{3} \bar{E}_{1}$ and $L_{2} L_{3} \bar{E}_{2}$. Let us look at $L_{2} L_{3} \bar{E}_{1}$ as an example (the same logic applies to all of them). In this case, the prompt lepton channel is $e \mu$ and, in addition, we get decays to $e \tau$. Since the tau will decay equally often to electrons and muons, we expect a large signal in the $e \mu$ channel both from the prompt leptons and the $e \tau$ channel. In addition we expect some contribution to the $e^{+} e^{-}$channel from the $e \tau$ decays, while $\mu^{+} \mu^{-}$ should give nothing. As can be seen from tables 3 and 4 this is, to a good approximation, indeed the case. We also notice that the dominance of the largest channel is quite similar for all the SPS points considered and all the four couplings. However, there is a clear difference between the two ways of measuring the branching fractions; for $L_{2} L_{3} \bar{E}_{1}$ we have $P_{e \mu} \approx 0.81$ while $\int \mathcal{N}_{e \mu} \approx 0.87$ and this difference is nearly independent of the choices for the SPS point and the R-violating coupling as well.

The estimate of the branching fraction of the dominant channel will depend strongly on which cuts are used as well as how many leptons not originating from neutralino decay we have. Therefore it is very difficult to make any precise predictions, but the difference seen between the two measures is expected due to the differences in the cuts used.

If we now compare the predictions from eq. (3.8) with the direct measurement of the fraction (3.7), we see that operators with an $e \tau$ channel give a measured fraction about twice as large as the prediction (3.8) (see $L_{1} L_{3} \bar{E}_{1}, L_{1} L_{3} \bar{E}_{3}$ and $L_{2} L_{3} \bar{E}_{1}$ in table 3), while for operators with a $\mu \tau$ channel the prediction (3.8) overestimates the fraction by about a factor two (see $L_{1} L_{3} \bar{E}_{2}, L_{2} L_{3} \bar{E}_{2}$ and $L_{2} L_{3} \bar{E}_{3}$ in table 3 ). This is in accordance with our expectation and illustrates that we can infer the presence of tau in the operator without having to rely on tau tagging. 


\begin{tabular}{|l|l|l|l|l|l|l|}
\hline model & $P_{e^{-} e^{+}}$ & $P_{\mu^{-} \mu^{+}}$ & $P_{\tau_{\text {jet }}^{-} \tau_{\text {jet }}^{+}}$ & $P_{e \mu}$ & $P_{e \tau_{\text {jet }}}$ & $P_{\mu \tau_{\text {jet }}}$ \\
\hline$L_{1} L_{2} \bar{E}_{1}$, SPS1a & 0.38 & 0.02 & 0.004 & 0.41 & 0.08 & 0.03 \\
$L_{1} L_{2} \bar{E}_{2}$, SPS1a & 0.02 & 0.40 & 0.004 & 0.40 & 0.03 & 0.07 \\
$L_{1} L_{2} \bar{E}_{3}$, SPS1a & 0.08 & 0.09 & 0.06 & 0.16 & 0.30 & 0.31 \\
$L_{1} L_{3} \bar{E}_{1}$, SPS1a & 0.44 & 0.003 & 0.03 & 0.10 & 0.36 & 0.02 \\
$L_{1} L_{3} \bar{E}_{2}$, SPS1a & 0.02 & 0.09 & 0.03 & 0.44 & 0.04 & 0.33 \\
$L_{1} L_{3} \bar{E}_{3}$, SPS1a & 0.09 & 0.02 & 0.26 & 0.10 & 0.40 & 0.13 \\
$L_{2} L_{3} \bar{E}_{1}$, SPS1a & 0.09 & 0.02 & 0.03 & 0.44 & 0.32 & 0.05 \\
$L_{2} L_{3} \bar{E}_{2}$, SPS1a & 0.005 & 0.45 & 0.03 & 0.10 & 0.02 & 0.35 \\
$L_{2} L_{3} \bar{E}_{3}$, SPS1a & 0.02 & 0.09 & 0.26 & 0.10 & 0.13 & 0.39 \\
\hline$L_{2} L_{3} \bar{E}_{1}$, SPS1a & 0.09 & 0.02 & 0.03 & 0.44 & 0.32 & 0.05 \\
$L_{2} L_{3} \bar{E}_{1}$, SPS1b & 0.09 & 0.01 & 0.04 & 0.44 & 0.35 & 0.04 \\
$L_{2} L_{3} \bar{E}_{1}$, SPS6 & 0.10 & 0.02 & 0.01 & 0.54 & 0.32 & 0.02 \\
\hline
\end{tabular}

Table 5. Branching fractions for different final states, estimated from events with 4 leptons (including $\left.\tau_{\text {jet}}\right)$ with a total charge-sum of zero.

\subsection{Redefining taus}

Another way of getting a grip on the decay channels containing taus is to include hadronic taus in the analysis; in this section leptons also include $\tau_{\text {jet }}$ 's and the only cuts imposed on the leptons are the acceptance cuts of $p_{T}>5 \mathrm{GeV}\left(10 \mathrm{GeV}\right.$ for $\left.\tau_{\text {jet }}\right)$ and isolation.

The event selection used here is exactly four leptons (including $\tau_{\text {jet }}$ 's), two positive and two negative. In addition, we require $p_{T}^{\text {miss }}>100 \mathrm{GeV}$.

We can then expand the definitions (3.3) and (3.5) to include $\tau_{\text {jet }}$ 's. Using these probabilities we can estimate all branching fractions of neutralinos decaying to lepton pairs. The result for all couplings at SPS1a is shown in table 5 along with the result for $L_{2} L_{3} \bar{E}_{1}$ at SPS1a, SPS1b and SPS6. It is clear from table 5 that this method does pick out the correct channels as the dominant ones; for all couplings, the two dominant ones are the same as those given in table 1 . It should be noted that channels with tau will give rise to several different final states depending on the decay of the tau. If we look at $L_{2} L_{3} \bar{E}_{1}$ at SPS6 the above points become clear; the two dominant final states are $e \mu$ and $e \tau_{\text {jet }}$ and the $e \tau$ channel contributes about $0.05-0.10$ to both $P_{e^{+} e^{-}}$and $P_{e \mu}$ and about 0.3 to $P_{e \tau_{\mathrm{jet}}}$ which is consistent with the branching ratios of taus. We also have some contamination of around 0.02 in all channels.

Compared to the cases of table 3, there are now more leptons that do not arise from neutralino decays. For example, for the SPS1a point, each of the couplings considered results in more $\tau_{\text {jet }}$ 's than expected from the neutralino decays alone. And when comparing the values for $L_{2} L_{3} \bar{E}_{1}$ at SPS1a, SPS1b and SPS6 we notice some differences which are consistent with the differences in the contributions from the cascade decay chain; e.g. we see that SPS6 gives fewer $\tau_{\text {jet }}$ 's than SPS1a and SPS1b. The conclusion is that the numbers are rather sensitive to the parameter point. 

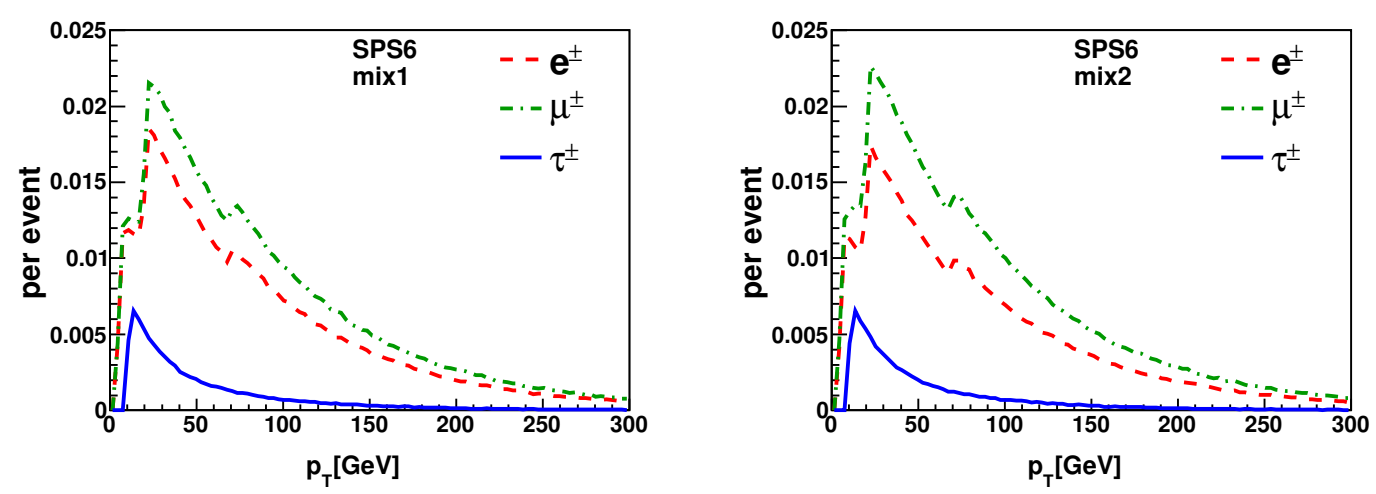

Figure 7. Electron, muon and $\tau_{\text {jet }}$ spectra for mix1 (left) and mix2 (right) at SPS6.

These issues make it hard to interpret the exact values of the branching fractions. It is also important to remember that tau tagging efficiencies should be added on top of this and that will further suppress the $\tau_{\text {jet }}$ 's in comparison with the light leptons.

\subsection{Combinations of couplings — case study}

To illustrate the usefulness of the observables discussed in the previous sections, let us study two cases where two operators are comparable, namely,

$$
\begin{array}{ll}
\operatorname{mix} 1: & L_{1} L_{2} \bar{E}_{1}=L_{2} L_{3} \bar{E}_{2}=10^{-4} ; \\
\operatorname{mix} 2: & L_{1} L_{2} \bar{E}_{2}=L_{2} L_{3} \bar{E}_{1}=10^{-4} .
\end{array}
$$

These combinations are chosen such that the total flavour content of mix1 and mix 2 are the same at the operator level. However, the effect of the cuts will be somewhat different and that will induce some differences. For example, the $p_{T}$ spectra for the two mixtures at SPS6 (as shown in figure 7) do look similar, but we can see some small differences caused by the fact that the light lepton flavour accompanying the tau gets suppressed by the cuts.

The corresponding invariant mass distributions (figure 8) are clearly different. For mix1 we see prompt di-lepton (i.e. eq. (B.2)) signals in each of the $e e, e \mu$ and $\mu \mu$ channels. We also see that at masses below some $100 \mathrm{GeV}$, the latter two distributions are slightly larger than the ee one (we ignore the triangular contributions from the cascade chain). A fit confirms that the difference is well explained by an additional contribution of type (B.11), which in turn suggests a $\mu \tau$ channel. It should be noted that the ability to detect a $\mu \tau$ channel in the light di-lepton distributions depends on the SUSY model so it cannot always be done, but we see that the $\mu \tau$ channel clearly shows up in the $\mu \tau_{\text {jet }}$ distribution as well.

Looking at mix 2 in the lower panels of figure 8 , we see a strong prompt $e \mu$ signal as well as a smaller prompt $\mu \mu$ signal. This is difficult to explain without assuming at least two couplings where both give $e \mu$ and only one gives $\mu \mu$. We also see a small signal in the $e e$ distribution, consistent with eq. (B.11) and the fit also prefers such a contribution to the $e \mu$ channel. This suggests an $e \tau$ channel, which is confirmed by the $e \tau_{\text {jet }}$ distribution. 

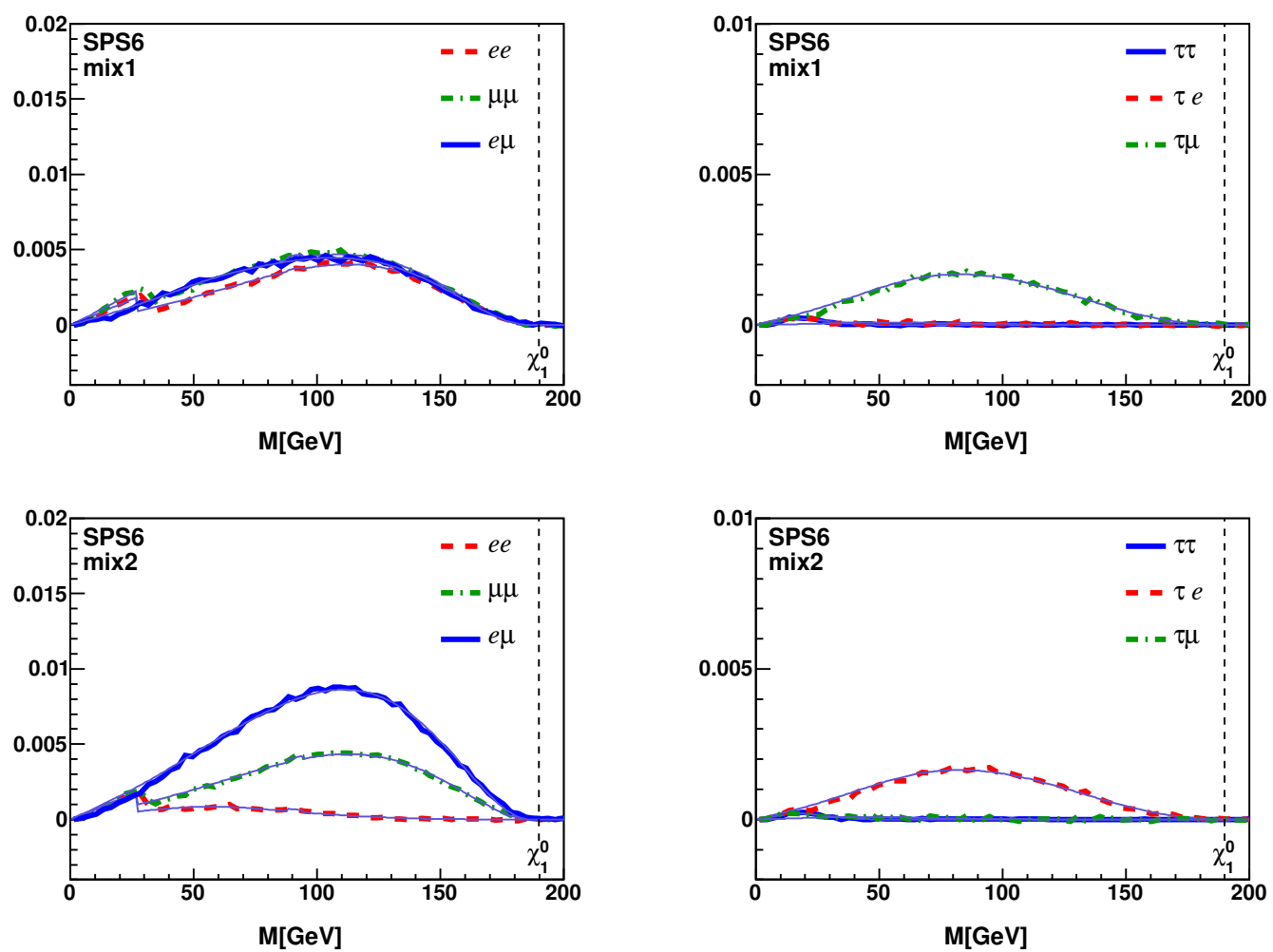

Figure 8. Di-lepton invariant masses for mix1 (upper panels) and mix2 (lower panels) at SPS6.

\begin{tabular}{|l|r|r|r|r|r|r|r|r|}
\hline model & $P_{e^{-} e^{+}}$ & $P_{e \mu}$ & $P_{\mu^{-} \mu^{+}}$ & $(3.7)$ & $(3.8)$ & $\int \mathcal{N}_{e e}$ & $\int \mathcal{N}_{e \mu}$ & $\int \mathcal{N}_{\mu \mu}$ \\
\hline mix1, SPS1a & 0.27 & 0.37 & 0.36 & 0.48 & 0.84 & 0.29 & 0.35 & 0.36 \\
mix1, SPS1b & 0.27 & 0.37 & 0.37 & 0.44 & 0.82 & 0.29 & 0.36 & 0.36 \\
mix1, SPS6 & 0.28 & 0.37 & 0.36 & 0.48 & 0.85 & 0.30 & 0.34 & 0.37 \\
\hline mix2, SPS1a & 0.07 & 0.62 & 0.30 & 1.31 & 0.63 & 0.05 & 0.63 & 0.31 \\
mix2, SPS1b & 0.08 & 0.62 & 0.29 & 1.47 & 0.65 & 0.05 & 0.64 & 0.31 \\
$\operatorname{mix}$, SPS6 & 0.08 & 0.62 & 0.31 & 1.35 & 0.63 & 0.06 & 0.62 & 0.32 \\
\hline
\end{tabular}

Table 6. Similar to table 3 for mix1 and mix2 at SPS1a, SPS1b and SPS6.

\begin{tabular}{|l|l|l|l|l|l|l|}
\hline model & $P_{e^{-} e^{+}}$ & $P_{\mu^{-} \mu^{+}}$ & $P_{\tau_{\text {jet }}^{-} \tau_{\text {jet }}^{+}}$ & $P_{e \mu}$ & $P_{e \tau_{\text {jet }}}$ & $P_{\mu \tau_{\text {jet }}}$ \\
\hline mix1, SPS1a & 0.19 & 0.24 & 0.02 & 0.25 & 0.05 & 0.21 \\
mix1, SPS6 & 0.23 & 0.29 & 0.003 & 0.29 & 0.01 & 0.17 \\
\hline mix2, SPS1a & 0.06 & 0.21 & 0.02 & 0.42 & 0.19 & 0.06 \\
mix2, SPS6 & 0.06 & 0.25 & 0.006 & 0.51 & 0.16 & 0.02 \\
\hline
\end{tabular}

Table 7. Similar to table 5 for mix1 and mix2 at SPS1a and SPS6. 
Let us now look at the counting observables from the last section. They are all listed in tables 6 and 7 . If we first look at table 6 , we again see that these numbers seem largely independent of the SPS point. When looking at mix1 we see that we have significant branching fractions to $e e, e \mu$ and $\mu \mu$ and that the last two are a little larger than the first. We also see that the measurement of the fraction (3.7) is about half the prediction from (3.8), suggesting a $\mu \tau$ channel which would also explain the higher values for $e \mu$ and $\mu \mu$ compared to $e e$.

If we instead look at mix2 we see a large branching fraction to $e \mu$ and also a significant $\mu \mu$ channel while the ee channel is small but non-zero. We also notice a measured fraction (3.7) that is twice that of the prediction (3.8), indicating an $e \tau$ channel, consistent with the small ee contribution.

If we finally turn our attention to table 7 , we see a larger variation with SPS point but the structure of the numbers is in agreement with our previous conclusions.

To summarize, the invariant mass distributions and the counting observables from the last section can independently help identify the relevant decay channels which then leads us to the correct combinations of operators. The comparison between the measurement of the fraction (3.7) and the prediction (3.8) is the most reliable way of detecting an $e \tau$ or $\mu \tau$ channel without relying on tau tagging.

\section{$4 \quad L Q \bar{D}$ coupling}

$L Q \bar{D}$ couplings can induce two types of neutralino decays. The neutralino can either go to one up-type and one down-type (anti)quark and a charged lepton or to a down-type quark-antiquark pair and a neutrino. In the case of $L_{i} Q_{3} \bar{D}_{k}$ the latter decay dominates since otherwise a top would have to be produced, which is either strongly suppressed by phase space or requires an off-shell top; hence, in these cases, there are no isolated charged leptons from the decay and the lepton flavor of the $\lambda^{\prime}$ operator cannot be determined.

Table 8 summarizes possible scenarios for the various flavour combinations. Operators easier to study are of the type $L_{1,2} Q_{1,2} \bar{D}_{3}$, where lepton identification combined with btagging might give some interesting signals. The reason the $\ell j$ signal is mentioned in table 8 is that in many cases the two jets can be so close in the detector that they merge into one jet and the peak expected in the $\ell j j$ distribution instead shows up in $\ell j$. This is also partially true for $\ell b_{\text {jet }}$; however, in that case a more realistic algorithm for b-tagging is needed to know how well the b-tagging works if the jets are so close that they might merge.

When the $\lambda^{\prime}$ operator has $\tau$ flavour the situation is rendered more complicated on account of the loss of information involving the neutrinos in the decay of the tau. The most problematic situation, however, appears when we have a $Q_{3}$ operator, as discussed above we only expect decays to a neutrino and two jets and therefore this scenario might be hard to identify.

\subsection{Event selection}

The task of suppressing backgrounds is a bit more difficult with $L Q \bar{D}$ couplings as compared to $L L \bar{E}$ couplings, especially when it comes to suppressing $t \bar{t}$ events. However, the lepton multiplicity is useful in this respect. 


\begin{tabular}{|l|l|l|}
\hline Coupling & Decay products & Signals \\
\hline$L_{1,2} Q_{1,2} \bar{D}_{1,2}$ & $\ell q \bar{q}, \nu q \bar{q}$ & $\ell j, \ell j j$ \\
$L_{1,2} Q_{1,2} \bar{D}_{3}$ & $\ell q \bar{b}, \nu q \bar{b}$ & $\ell b_{\text {jet }}, \ell b_{\text {jet }} j$ \\
$L_{3} Q_{1,2} \bar{D}_{1,2}$ & $\tau q \bar{q}, \nu q \bar{q}$ & $\tau_{\text {jet }} j, \tau_{\text {jet }} j j$ \\
$L_{3} Q_{1,2} \bar{D}_{3}$ & $\tau q \bar{b}, \nu q \bar{b}$ & $\tau_{\text {jet }} b_{\text {jet }}, \tau_{\text {jet }} b_{\text {jet }} j$ \\
$L_{1,2,3} Q_{3} \bar{D}_{1,2}$ & $\nu b \bar{q}$ & $b_{\text {jet }} j$ \\
$L_{1,2,3} Q_{3} \bar{D}_{3}$ & $\nu b \bar{b}$ & $b_{\text {jet }} b_{\text {jet }}$ \\
\hline
\end{tabular}

Table 8. The various possible scenarios with $L Q \bar{D}$ operators. It is here assumed that the neutralino is lighter than the top. Notation: $\tau_{\text {jet }}, b_{\text {jet }}$ and $j$ denote a tau jet, a b-jet and a light-flavor jet, respectively.

To cover most possibilities we use three different cuts in this analysis. All three require:

- Transverse sphericity $>0.2$,

- $E_{T}=\Sigma_{\text {leptons,jets }}\left|p_{T}\right|>1000 \mathrm{GeV}$,

- Hardest jet $p_{T}>300 \mathrm{GeV}$.

We define a "hard cut" to additionally require at least two same-sign isolated leptons with $p_{T}>20 \mathrm{GeV}$. The same-sign requirement suppresses the $t \bar{t}$ background by several orders of magnitude, whereas the signal is reduced to only about $1 \%$ of its precut value.

An alternative is to use a "loose cut" which passes a lot more of the signal, at the cost of a significant background from $t \bar{t}$ events. This requires only one isolated lepton with $p_{T}>20 \mathrm{GeV}$, and at least 7 jets with $p_{T}>20 \mathrm{GeV}$.

The third cut is meant for the cases where we only get neutrinos and no leptons. Therefore it requires instead of leptons, $p_{T}^{\text {miss }}>200 \mathrm{GeV}$, and at least 7 jets with $p_{T}>20 \mathrm{GeV}$. We name this the "pTmiss cut".

The effects of these cuts, on some representative $L Q \bar{D}$ couplings as well as on the $t \bar{t}$ background, are summarized in table 9 . All other backgrounds are less important than $t \bar{t}$ for these cuts.

We see that for the hard cut we get a satisfactory suppression of the $t \bar{t}$ while the loose cut will suffer from a significant contamination by $t \bar{t}$ events.

It is also clear that the operators with tau flavour are more problematic due to fewer leptons. As expected, of the operators in table 9, the most problematic one is $L_{1} Q_{3} \bar{D}_{3}$ where the signal would be $\nu_{e} b b$ or $e^{-} t b$. With the $t$-quark above the kinematical threshold, only the $\nu_{e} b b$-channel is open and we have to rely on the pTmiss cut. This last case will appear very similar to an R-parity conserving scenario, the most significant difference being that the particles now responsible for the missing $p_{T}$ are the (massless) neutrinos rather than the neutralinos. This problem is present for all operators of type $L Q_{3} \bar{D}$. 


\begin{tabular}{|l|l|l|l|}
\hline model & hard cut & loose cut & pTmiss cut \\
\hline$L_{1} Q_{1} \bar{D}_{2}$, SPS1a & 0.011 & 0.082 & 0.043 \\
$L_{1} Q_{1} \bar{D}_{2}$, SPS1b & 0.043 & 0.24 & 0.16 \\
$L_{1} Q_{1} \bar{D}_{2}$, SPS3 & 0.06 & 0.25 & 0.16 \\
$L_{1} Q_{1} \bar{D}_{2}$, SPS5 & 0.015 & 0.089 & 0.046 \\
$L_{1} Q_{1} \bar{D}_{2}$, SPS6 & 0.008 & 0.096 & 0.083 \\
\hline$L_{1} Q_{1} \bar{D}_{2}$, SPS1a & 0.011 & 0.082 & 0.043 \\
$L_{1} Q_{3} \bar{D}_{3}$, SPS1a & 0.0013 & 0.03 & 0.048 \\
$L_{3} Q_{1} \bar{D}_{3}$, SPS1a & 0.0027 & 0.043 & 0.041 \\
\hline$t \bar{t}$ & $1.5 \cdot 10^{-6}$ & 0.002 & 0.00083 \\
\hline
\end{tabular}

Table 9. The fraction of events that passes the various cuts for some $L Q \bar{D}$ operators, as well as $t \bar{t}$ production. Note that this does not take the different cross-sections into account.

\subsection{Invariant mass distributions}

There are plenty of interesting invariant mass distributions to be studied with respect to $L Q \bar{D}$ operators. Unfortunately, the lack of knowledge of the charge of light quark jets implies that the same-sign subtraction tool is essentially rendered inoperative. As a result, most signals tend to drown in combinatorial and other backgrounds. This problem would be reduced if the couplings were small enough for displaced vertices to appear, however, as mentioned before, we do not make use of such possibilities here.

A further problem is that the boost of the neutralino results in all the decay products being very close in the detector, thereby raising the probability of jets merging. It is, therefore, not always true that we get two jets and one lepton from each neutralino. On the other hand, the closeness of the decay products can be used to choose which jets and leptons to compute the invariant mass of.

These proximity issues make the choice of jet algorithm very important. Especially the parameter $R_{\text {jet }}$ is very important. To optimize each analysis, we perform it using jets as defined by $R_{\text {jet }}$ equal to $0.2,0.4$ and 0.7 and show results using the most favourable value for each analysis. The event selection always uses jets as defined when $R_{\text {jet }}=0.4$.

In figure 9 these points can be seen for $L_{1} Q_{1} \bar{D}_{2}$ at SPS1a: if $\ell j j$ where the two jets are within $\Delta R=1$ from the lepton, are used, one can get a very nice peak at the neutralino mass (black curve, upper left panel). If all $\ell j j$ are used, the peak is less pronounced but can be clearly seen both with $R_{\text {jet }}=0.4$ as in the lower left panel, and even better when $R_{\text {jet }}=0.2$, which can be seen in the upper right panel. Since the two quark jets quite often merge into one, it is also possible to see a nice peak at the neutralino mass in the distribution of all $\ell j$ combinations, as seen in the upper left panel. It is also worth noting that the branching fraction for neutralino decay to $\ell j j$ as compared to the fraction to $\nu j j$ depends on the SUSY parameter point and e.g. SPS1b has a more favourable value, which can be seen in the lower right panel of figure 9, where the peak is clearly more pronounced than in the comparable plot for SPS1a (lower left panel). 

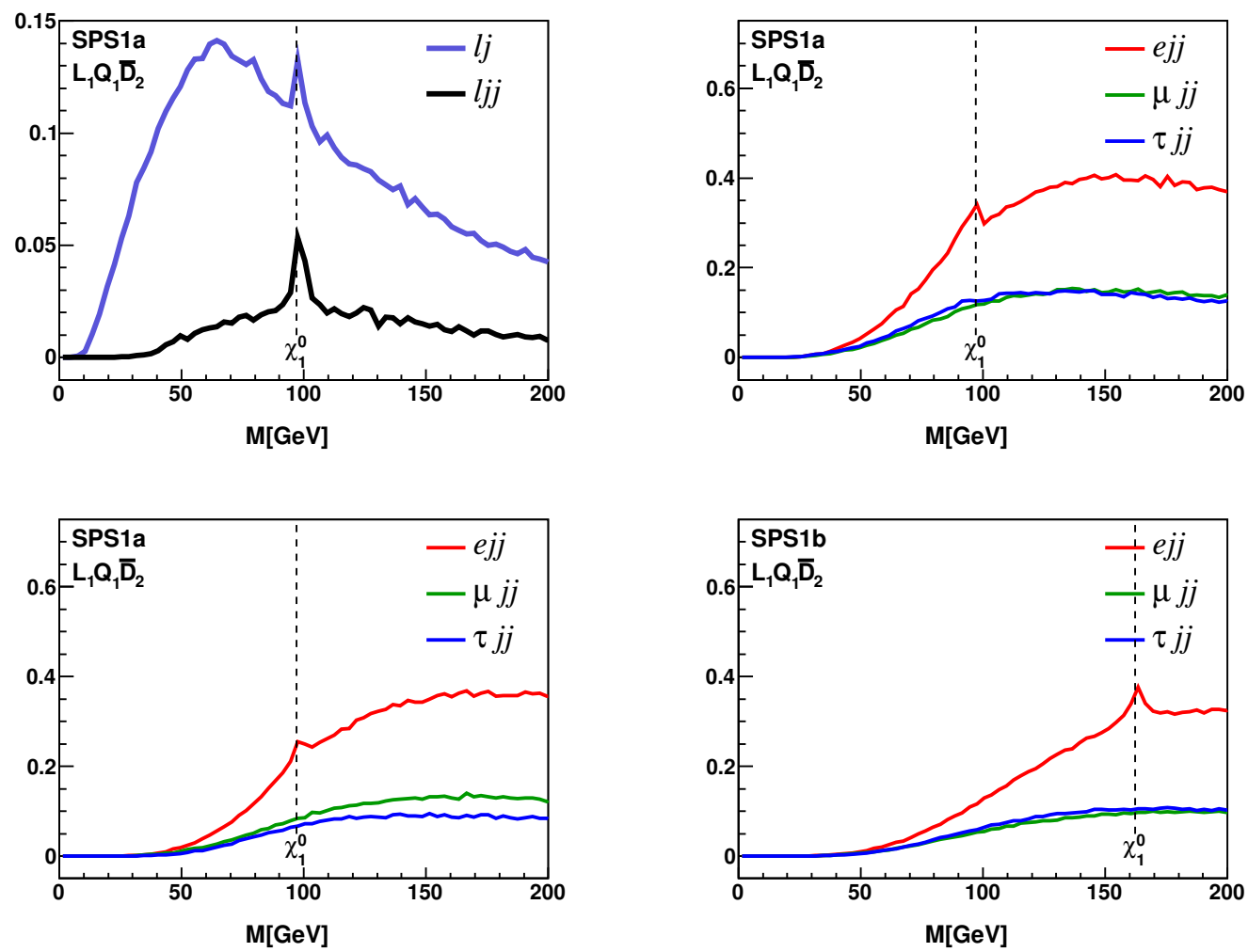

Figure 9. Invariant mass distribution for $L_{1} Q_{1} \bar{D}_{2}$ at SPS1a: upper left: violet: $\ell j$ distribution, black: $\ell j j$ where the jets are closer than $\Delta R=1$ to the lepton, this distribution has been multiplied by 5 to be visible. Upper right: $\ell j j$ distributions using $R_{\text {jet }}=0.2$. Lower left panel: same as upper right but with $R_{\text {jet }}=0.4$, lower right: same as lower left but for SPS1b. In this figure, the hard cut has been used.

So far we have discussed the case with one light lepton and two light jets in the decay. Another group of operators have b-quarks in the decay and that opens up for b-tagging. Assuming that the b-tagging can also determine the charge of the $b_{\text {jet }}$, it becomes possible to use same-sign subtraction to reduce the combinatorial background.

Figure 10 shows $\ell b_{\text {jet }} j$ distributions as well as $\ell b_{\text {jet }}$ distributions where same-sign subtraction has been employed on the $\ell b_{\text {jet }}$ pair. In the upper left panel we see that this gives a good signal for $L_{1} Q_{1} \bar{D}_{3}$ where we again have a peak at the neutralino mass. The signal in the $e b_{\text {jet }}$ channel looks like the distribution (B.2) with a small peak at the high end (caused by merged jets), which is consistent with our expectation. Due to the low number of charged leptons in the neutralino decay at SPS1a, we have used the loose cut to achieve the upper panels of figure 10. For SPS1b we get more charged leptons and as is clearly seen in the middle panels the hard cut is fine for extracting these signals. However, the SUSY production at SPS1b is also about one order of magnitude smaller than at SPS1a so using the hard cut is more important to reduce the backgrounds.

If the lepton is a $\tau_{\text {jet }}$, things are much more difficult; first of all we lose energy to the neutrino, so we do not expect a peak anymore. However, if we use $R_{\text {jet }}=0.2$ together 

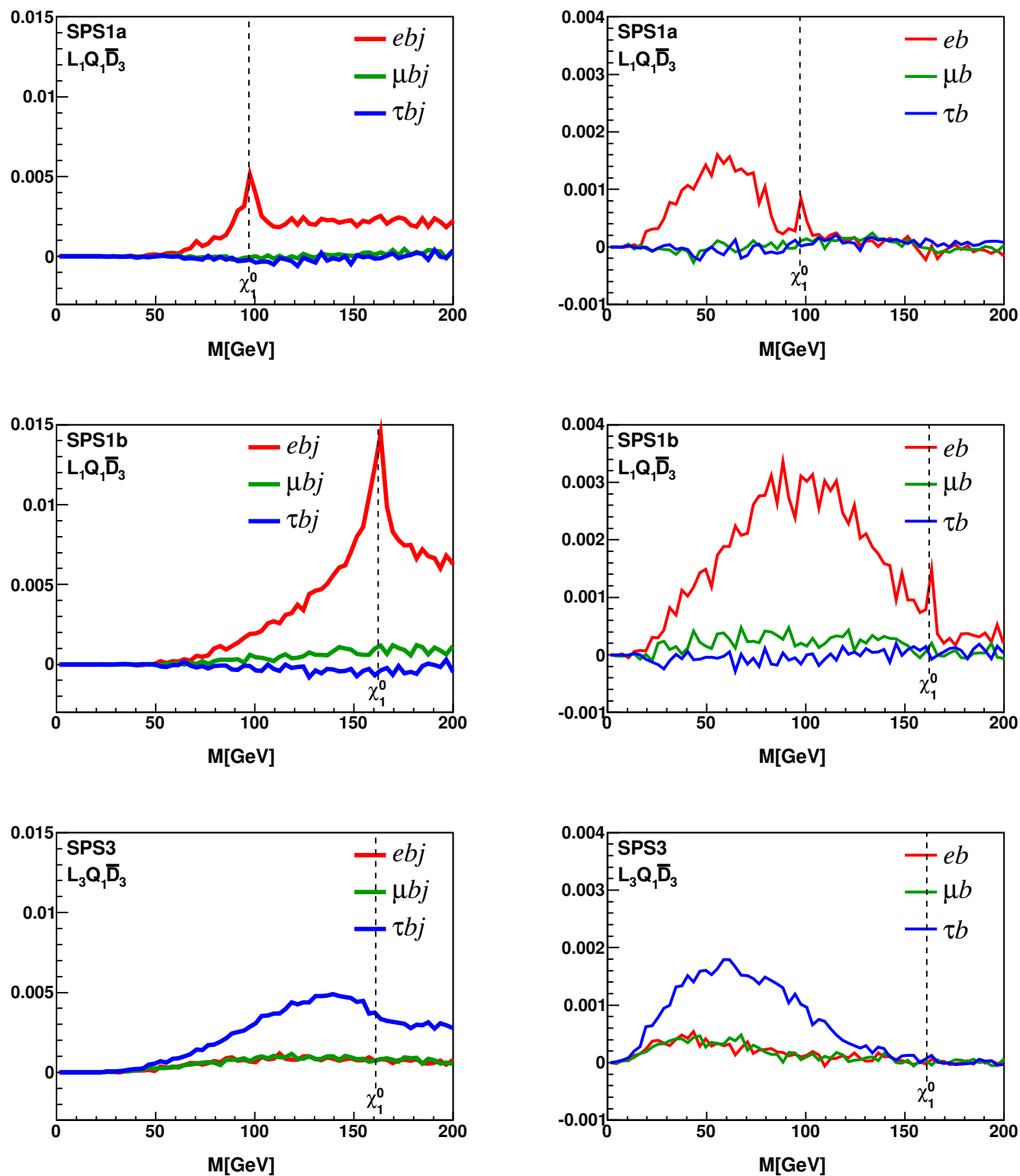

Figure 10. Left panels: $\ell b_{\text {jet }} j$ distributions with same-sign subtraction in the $\ell b_{\text {jet }}$ pair. Right panels: $\ell b_{\text {jet }}$ distributions with same-sign subtraction. Upper and middle panels: $L_{1} Q_{1} \bar{D}_{3}$ at SPS1a (upper) using the loose cut and SPS1b (middle) using the hard cut. Lower panels: $L_{3} Q_{1} \bar{D}_{3}$ at SPS3 with $R_{\text {jet }}=0.2$ and using the pTmiss cut.

with the pTmiss cut for SPS3 we see that the expected signals are clearly there. In the $\tau_{\text {jet }} b_{\text {jet }} j$ distribution (lower left panel) we see a bump below the neutralino mass. Perhaps the best and most conclusive signal though, is in the $\tau_{\text {jet }} b_{\text {jet }}$ channel (lower right channel) where we see a distribution similar to what we discussed in appendix B for tau decays (the $\ell \tau_{\text {jet }}$ distribution of figure 21 ). 

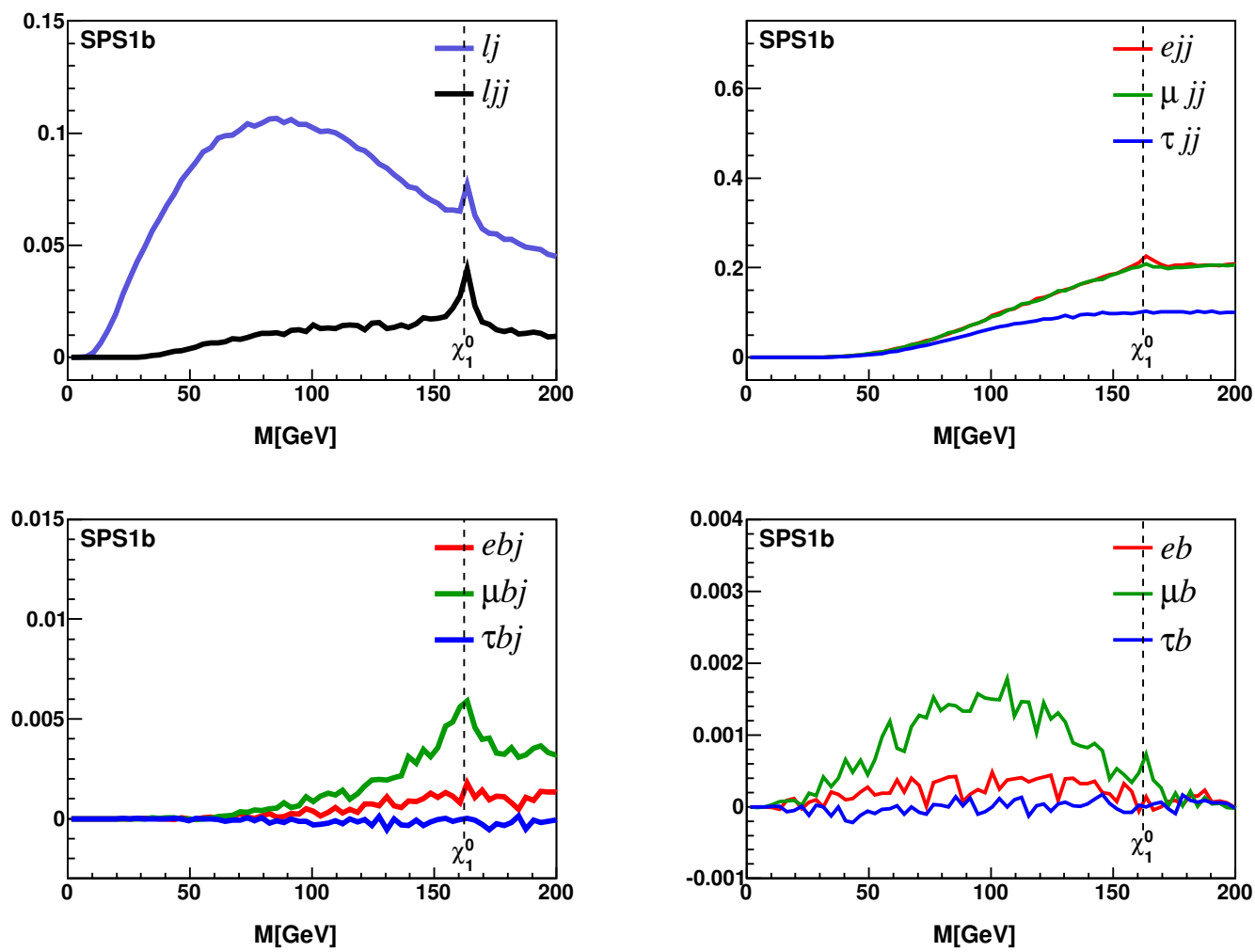

Figure 11. Combinations of $L Q \bar{D}$ operators: Invariant masses for $\lambda_{121}^{\prime}=10^{-4}$ and $\lambda_{223}^{\prime}=10^{-4}$ at SPS1b. The two operators differ both in lepton flavours and in quark flavours. Upper left: $\ell j$ and $\ell j j$ (magnified by a factor 5), in the latter case the two jets are close to the lepton (see figure 9). Upper right: $\ell j j$. Lower panels show $\ell b_{\text {jet }} j$ (left) and $\ell b_{\text {jet }}$ (right) distributions. All plots use the hard cut.

\subsection{Mixing $L Q \bar{D}$ operators}

The chances to identify two or more simultaneously large $L Q \bar{D}$ type operators, depend strongly on the specific combination; if the two operators are identified through different channels, the identification can be rather straight-forward.

One example of this can be seen in figure 11 where one operator gives a signal in $\mu b_{\text {jet }} j$ and $\mu b_{\text {jet }}$ channels and the other shows up in the ejj distribution. This case is rather fortunate since the two operators can be identified independently; if the lepton flavour of the operators would have been the same, it would have been much harder to tell that we have one operator with and one without a $\bar{D}_{3}$ component.

In figure 12 we show another combination where an operator $\left(\lambda_{211}^{\prime}\right)$ giving a peak in the $\mu j j$ distribution, is combined with a more problematic tau-flavour coupling $\left(\lambda_{313}^{\prime}\right)$ and again we see that both operators can be identified successfully.

In conclusion, having more than one large $L Q \bar{D}$ operator at the same time is no problem as long as the signals from the couplings appear in different channels and the operators are identifiable on their own. 

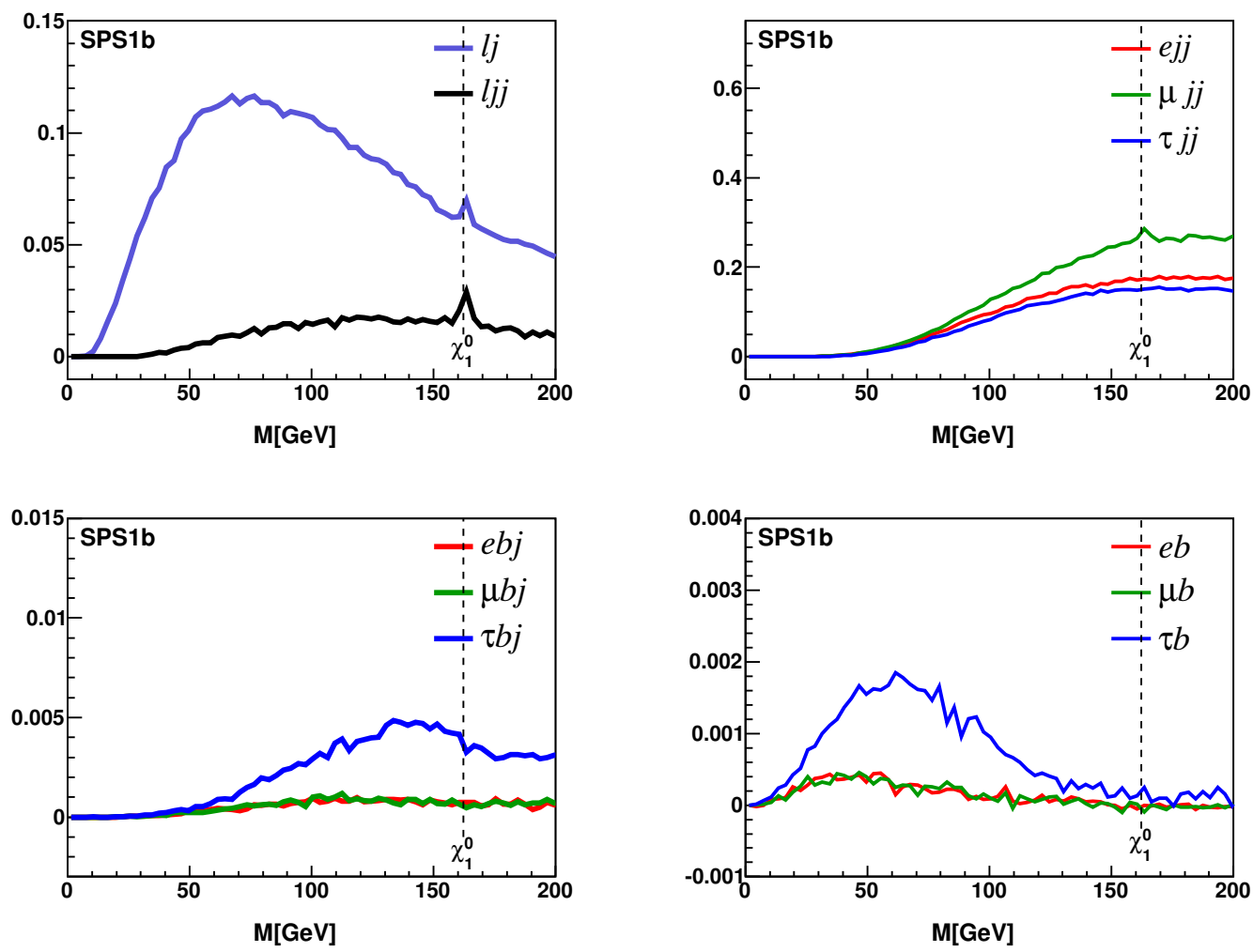

Figure 12. Same as figure 11 for $\lambda_{211}^{\prime}=10^{-4}$ and $\lambda_{313}^{\prime}=10^{-4}$ at SPS1b. Here the two lower panels use the pTmiss cut as well as $R_{\text {jet }}=0.2$ to get enough $\tau_{\text {jet }}$ 's.

\subsection{Backgrounds}

As mentioned before, the most important background is $t \bar{t}$. Although the hard cut is very effective in suppressing this background; the need to sometimes use the loose or pTmiss cut makes it necessary to look at the expected invariant mass distributions from the $t \bar{t}$ events.

The interesting distributions here are the invariant masses including $b_{\text {jet }}$ since that is where the weaker signals occur and we expect some contribution there from $t \bar{t}$ due to tops decaying to $\ell b_{\text {jet }}(\nu)$.

These distributions are shown in figure 13 where we can see that the only significant distribution is in the $\mu b_{\text {jet }}$ and $e b_{\text {jet }}$ channels for the loose cut. The distribution can be understood as a triangular distribution ending around $150 \mathrm{GeV}$ from the decay of the top and a combinatorial background that due to the same-sign subtraction and the charge correlation between the top and antitop, is negative.

It is also important to note that we see no significant signal when the pTmiss cut and $R_{\text {jet }}=0.2$ are used. Simulations where standard model processes were added, also show that the studied distributions are not significantly changed by these backgrounds. 

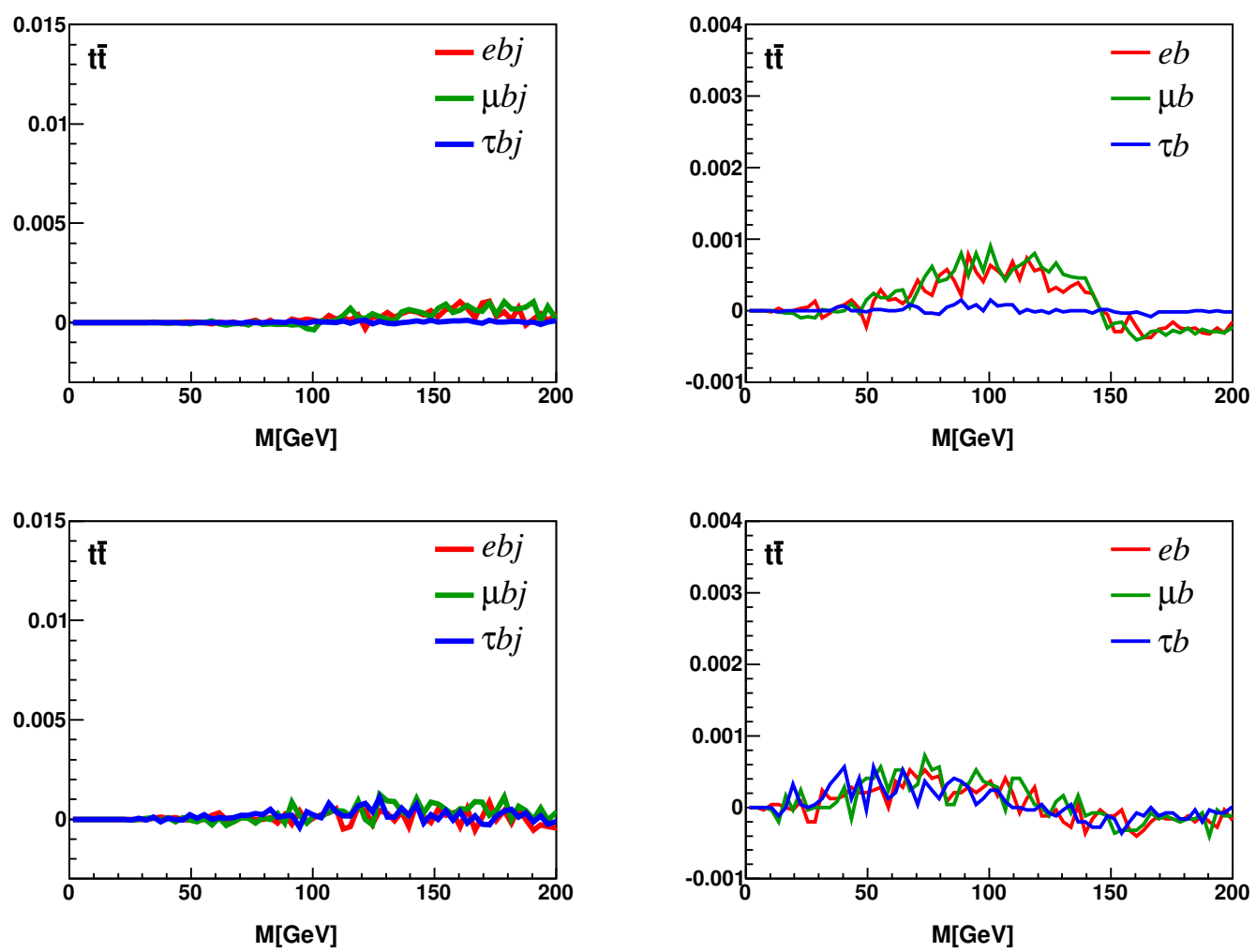

Figure 13. Invariant mass distributions for $t \bar{t}$ events. The left panels show $\ell b_{\text {jet }} j$ distributions and the right panels show $\ell b_{\text {jet }}$ distributions. In the upper panels the loose cut is used and in the lower panels the pTmiss cut is used and $R_{\text {jet }}=0.2$.

\section{$5 \quad \bar{U} \bar{D} \bar{D}$ coupling}

Of the various scenarios with trilinear R-parity violation, the one that is most difficult to analyse at a hadronic collider is that of non-zero $\bar{U} \bar{D} \bar{D}$ couplings. With the lightest neutralino now decaying into three jets, extracting the signal from the large QCD background is very challenging [64]. In fact, even for simpler situations like resonance production or one-step decays, the reach of the Tevatron or the LHC is very limited [30, 31, 65].

As mentioned earlier, assuming moderately light neutralinos, we expect them to be highly boosted due to the large center of mass energy of the collision. This means that the decay products of the neutralino will be rather collimated, which has enormous implications for the identification of $L Q \bar{D}$ operators as discussed in the previous section. This would also be the case for $\bar{U} \bar{D} \bar{D}$ operators where the three jets from the decaying neutralino will sometimes be so collimated that they merge. This very feature can be turned to an advantage; a $\bar{U} \bar{D} \bar{D}$ signal may be extracted by studying jet substructure, where one looks for a big jet that looks like it could consist of three jets that have merged [66].

There is, however, an exception to the above picture, namely any of the three operators $\bar{U}_{3} \bar{D}_{i} \bar{D}_{j}$, for the top flavour of these couplings ensures a very different behavior. If the neutralino is lighter than the top, it may appear stable on the detector scale and 


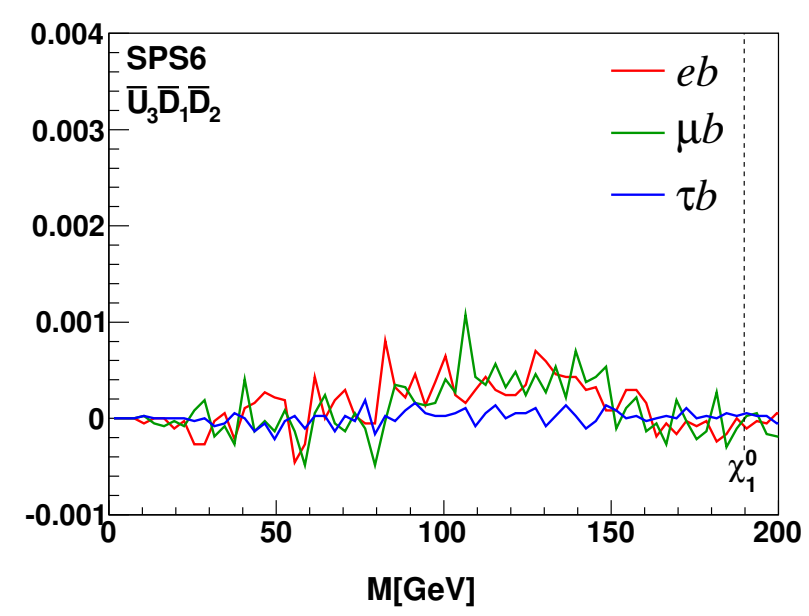

Figure 14. Invariant mass distributions for $\lambda_{312}^{\prime \prime}$ at SPS6. The distributions shown are $\ell b_{\text {jet }}$ distributions for all lepton flavours.

we might be facing a fake MSSM scenario. On the other hand, if the neutralino is heavier than the top, it decays into a top (or antitop) and two jets that often would tend to be soft (due to limited phase space). While a $t \bar{t}+n$-jets final state has a very large QCD background, note that the latter decreases fast with both increasing $n$ as well as with an increasing $p_{T}^{\min }$ (jet) (the minimal requirement on the transverse momenta of these additional putative jets). And as additional jets accrue not only from the decay of the neutralinos, but also in earlier steps in the SUSY cascade, and as at least some of them are likely to have a sufficiently large $p_{T}$, this final state is worth investigating. However, rather than performing this admittedly difficult task, we recommend concentrating on a relatively cleaner subsample. Noting that the neutralinos are Majorana particles, the very last pair of decays is as likely to produce a like-sign top-pair ( $t t$ or $\overline{t t}$ ) as an opposite-sign one. The possibility of same-sign tops makes the extraction of these events rather straight forward. One way is to look for same-sign isolated leptons accompanied by two same-sign b-jets; this approach was taken in a similar study [67].

However, it turns out that the harder cuts used on $L Q \bar{D}$ operators are also efficient in this case. This comes as no surprise since those cuts are based on same-sign leptons. If we, for example, look at $\bar{U}_{3} \bar{D}_{1} \bar{D}_{2}$ at SPS6 where the neutralino mass is $189 \mathrm{GeV}$ so that the top decay channel is open, about $1 \%$ of the events pass the hard cut from the $L Q \bar{D}$ section. This leaves good hope for detection and if we then look at the $\ell b_{\text {jet }}$ invariant mass, as is done in figure 14, we see the same structure (although rather weak) as in the corresponding distribution from $t \bar{t}$ events as shown in the upper right panel of figure 13. This similarity demonstrates the presence of top quarks in the events and can therefore be used to confirm the presence of a $\bar{U}_{3} \bar{D}_{j} \bar{D}_{k}$ operator. 

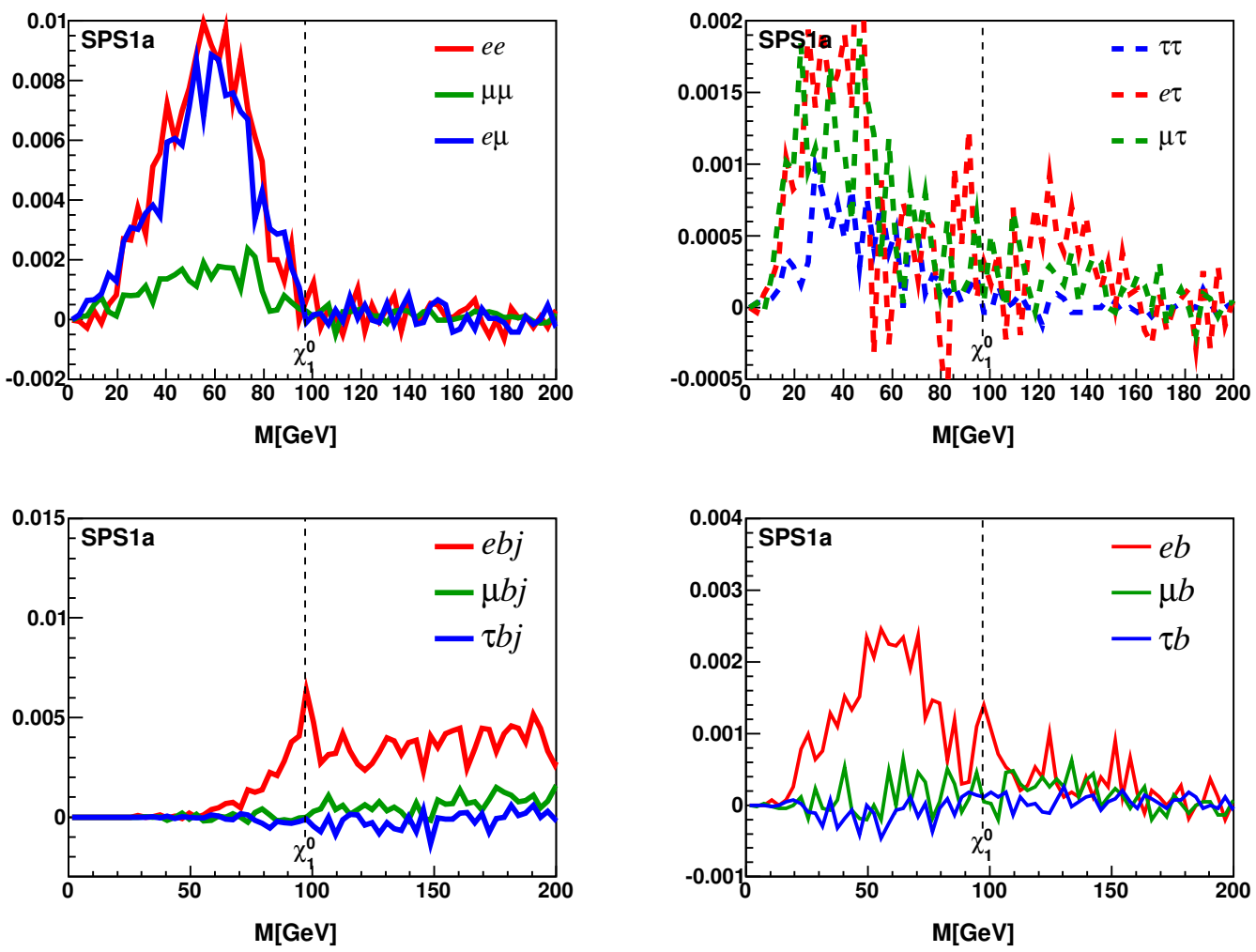

Figure 15. Mix of operators: Invariant mass distributions for $\lambda_{121}=10^{-5}$ and $\lambda_{123}^{\prime}=10^{-4}$ at SPS1a. Upper panels: di-lepton invariant mass distributions. Lower left panel shows $\ell b_{\text {jet }} j$ and lower right shows $\ell b_{\text {jet }}$.

\section{$6 \quad$ Mixing and models}

To date, most phenomenological analyses have assumed the dominance of a single Rviolating operator. However, once a flavour structure is invoked (e.g., to explain fermion masses and mixings), ${ }^{8}$ hierarchies in R-violating couplings would be related to the flavour charges of the respective fields [37-41]. Even more importantly, even if only one R-violating operator is to be postulated in the interaction basis, fermion mixing would, in general, induce non-zero values for others. This implies that it is natural to expect a range of hierarchies for R-violating operators.

\section{1 $L L \bar{E}$ and $L Q \bar{D}$ operators}

Before entering into a discussion of models that require operators being related in magnitude, we shall first look at some examples of the presence of two different operators. Without jeopardizing proton stability, we can allow for the simultaneous presence of $L L \bar{E}$ and $L Q \bar{D}$ operators.

\footnotetext{
${ }^{8}$ See e.g. [68].
} 
Since both $L Q \bar{D}$ and $L L \bar{E}$ operators can be identified due to the lepton multiplicity, the presence of one might mask the presence of the other, especially if the corresponding decay widths are comparable. Interestingly, for many of the SPS points in question, having one each of $L L \bar{E}$ and $L Q \bar{D}$ operators of the same magnitude, results in the decay width associated with the $L L \bar{E}$ coupling being about $10-20$ times larger than that due to the $L Q \bar{D}$ operator. ${ }^{9}$ Furthermore, even if the decay widths for the two sets of channels were comparable, the $L L \bar{E}$ ones would be more visible due to their higher lepton multiplicity which implies easier detection. Thus, in order to study scenarios where one might actually see effects of both operators at the same time, we take the $L Q \bar{D}$ operator to be 10 times larger than the $L L \bar{E}$ one.

While most of the conclusions from the study of single couplings are still valid when more than one coupling is relevant, the details of section 3.6 are modified. In particular, the differences between the measured and estimated values of the fraction (3.7) no longer point to the relevant tau channels. Therefore, it is more useful to study invariant mass distributions.

A favourable scenario would be the combination of two operators that are both relatively easy to identify. One example of this would be $\lambda_{121}=10^{-5}$ and $\lambda_{123}^{\prime}=10^{-4}$. The invariant mass distributions corresponding to this scenario, at SPS1a, are shown in figure 15. We clearly see the signals expected from the $L L \bar{E}$ coupling in the $e \mu$ and $e e$ distributions (upper left panel) as well as the $\ell b_{\text {jet }} j$ (lower left panel) signals expected from the $L Q \bar{D}$ coupling. We also note that the distributions including $\tau_{\text {jet }}$ 's (in the upper right plot) show signals that might suggest a $\tau \tau$ channel, however, those tau pairs actually come from the cascade chain and one has to be careful not to interpret them as decay products of the neutralino.

Whether the potentially confusing background from the cascade is present or not, depends on the parameter point, in e.g. SPS6 it is not. However, SPS6 comes with another problem; it turns out that the branching fractions for decay to charged leptons plus quarks is much smaller (factor $\approx 7$ ) than the branching fraction to neutrino plus quarks. This, then, results in fewer events with leptons and, as a consequence, the identification of the $L Q \bar{D}$ operator is rendered more difficult.

Similarly, for other experimentally complicated scenarios, such as operators with a lot of tau flavour, the problems (in identifying the $L Q \bar{D}$ operator) noted in section 4 remain pretty much unchanged when combinations are considered. In general, the identification of the $L L \bar{E}$ and the $L Q \bar{D}$ operators can be handled independently and the only interference between them is that for the smaller coupling we get less statistics to work with. This also means that there is an interval where both operators can be identified while in the periphery we will see only one dominant operator. This interval is centered roughly where the $L Q \bar{D}$ operator is about 10 times larger than the $L L \bar{E}$ coupling. In general, the $L L \bar{E}$ operators are easier to identify, which allows for smaller $L L \bar{E}$ couplings to be successfully identified.

\subsection{Link with flavour symmetries: two examples}

\footnotetext{
${ }^{9}$ The reason for this is not far to seek. The squarks tend to be much heavier than the sleptons, and, of the latter, the stau tends to be the lightest. Consequently, the purely leptonic decay modes of the LSP dominate over the semileptonic ones.
} 


\begin{tabular}{|c|ccccccc|}
\hline & $Q_{i}$ & $\bar{U}_{i}$ & $\bar{D}_{i}$ & $L_{i}$ & $\bar{E}_{i}$ & $H_{2}$ & $H_{1}$ \\
\hline $\mathrm{U}(1)$ & $a_{i}$ & $a_{i}$ & $a_{i}$ & $b_{i}$ & $b_{i}$ & $-2 a_{3}$ & $-2 a_{3}$ \\
\hline
\end{tabular}

Table 10. Assignments of $\mathrm{U}(1)$ charges.

\begin{tabular}{|c|ccccc|}
\hline$i j k$ & 111 & 121 & 122 & 222 & 131 \\
\hline $\mathrm{U}(1)$ & $-12-w$ & $-7-w$ & $-2-w$ & $3-w$ & $-8-w$ \\
\hline$i j k$ & 133 & 333 & 223 & 233 & 123 \\
\hline $\mathrm{U}(1)$ & $-4-w$ & $-w$ & $2-w$ & $1-w$ & $-3-w$ \\
\hline
\end{tabular}

Table 11. Operator charges in a model (see text) with both family and Left-Right symmetry. Here $w$ parametrises flavour-independent contributions [42].

For a given flavour model, the relative magnitude of R-violating operators is, to a large extent, predicted, allowing us to tabulate the expected signals in neutralino decays. This will be done for two representative cases, namely Left-Right symmetric models and SU(5).

\subsubsection{Left-right-symmetric models}

The simplest starting point is a single $\mathrm{U}(1)$ family symmetry with the same charges for the left- and right-handed states (left-right symmetry) as shown in table 10, where, e.g., the choice $a_{i}=(-4,1,0)[3,4]$ gives an acceptable pattern for the mass matrices.

With these charge assignments the quark mass matrices (up to numerical factors and phases, which, in general, are expected to be of order unity) take the form

$$
M^{\mathrm{up}} \sim\left(\begin{array}{ccc}
\epsilon^{8} & \epsilon^{3} & \epsilon^{4} \\
\epsilon^{3} & \epsilon^{2} & \epsilon \\
\epsilon^{4} & \epsilon & 1
\end{array}\right), \quad M^{\text {down }} \sim\left(\begin{array}{ccc}
\bar{\epsilon}^{8} & \bar{\epsilon}^{3} & \bar{\epsilon}^{4} \\
\bar{\epsilon}^{3} & \bar{\epsilon}^{2} & \bar{\epsilon} \\
\bar{\epsilon}^{4} & \bar{\epsilon} & 1
\end{array}\right)
$$

where $\bar{\epsilon} \approx \sqrt{\epsilon} \approx 0.2$.

We now consider the effect of the U(1) symmetry on the pattern of allowed $R$-violating interactions [37-41]. In this simple example, with all fermions of a given family having the same charge and with a left-right symmetry, the charges of the operators depend only on the combination $(i, j, k)$ and are independent of the type, viz. $L L \bar{E}, L Q \bar{D}$ or $\bar{U} \bar{D} \bar{D}$, (see table 11).

The parameter $w$ accounts for the fact that the charge assignment is not unique in model constructions [42] (although it is strongly constrained by phenomenological and theoretical arguments). For instance, while the addition of flavour independent contributions would not modify the fermion mass and mixing ratios, it can affect other considerations, such as anomaly cancellation conditions. Flavour-independent contributions could also arise from additional fields with a non-trivial flavour charge that couple to all operators. These issues in the context of R-violating hierarchies are addressed in more detail in ref. [42].

The above flavour symmetries cannot ensure, by themselves, that rapid proton decay is avoided. This is done by imposing a baryon or a lepton parity $[3-5,34]$, which imply that 
the cases with $\Delta L \neq 0$ and with $\Delta B \neq 0$ have to be considered separately. For instance, lepton-number violating operators can be eliminated by imposing a lepton triality [34], under which the fields transform as

$$
Z_{3}:\left(Q, \bar{U}, \bar{D}, L, \bar{E}, H_{1}, H_{2}\right) \rightarrow\left(1,1,1, a, a^{2}, 1,1\right) .
$$

This allows only the baryon-number-violating operators and the mass terms, while forbidding lepton-number-violating ones. On the other hand, to forbid baryon-number violating operators, we would work instead with a baryon triality, such as in ref. [3, 4], viz.

$$
Z_{3}:\left(Q, \bar{U}, \bar{D}, L, \bar{E}, H_{1}, H_{2}\right) \rightarrow\left(1, a^{2}, a, a^{2}, a^{2}, a^{2}, a\right) .
$$

Along these lines, we can use the hierarchies implied by table 11 to make the link between flavour models and the expected decay rates in neutralino decays for two separate cases, namely:

- for $L L \bar{E}$ and $L Q \bar{D}$ operators;

- for $\bar{U} \bar{D} \bar{D}$ operators.

While $w$ can be adjusted in order to ensure that all operators remain within the experimental bounds, there can be additional sources of suppression in the couplings (arising due to a small $\tan \beta$ in supersymmetric models, the form of the Kähler potential, or additional, model dependent, features of the theory that may involve extra fields and symmetries). To keep the problem manageable (especially since our purpose here is to show how specific models can be tested in flavour neutralino decays), we will:

- assume that the couplings that correspond to higher flavours are bigger (have smaller flavour charge). The Yukawa couplings generating fermion masses are larger for the higher generations, having a smaller net flavour charge. If this is true for R-violating couplings as well, then the operators that involve third-generation flavours would dominate also in this case. Dominance of the R-violating couplings of the heavier flavours would also favour radiative over three body gravitino decays in scenarios with gravitino dark matter [33, 34].

- ensure that all couplings remain within the experimental bounds.

For $\Delta L \neq 0$ the strictest bounds are on $L_{1} Q_{1} \bar{D}_{1}$ from nuclear $\beta \beta$ decay and on $L_{1} L_{3} \bar{E}_{3}$ from bounds on Majorana neutrino masses, constraining the choice of the charge $w$ to be $|12+w| \geq 2$ and $|4+w| \geq 2$, which are easy to satisfy [42]. We also note that the magnitudes of the couplings in table 11 are symmetric in the three indices $i j k$. This implies, for example, that, at this level, the $\lambda_{121}^{\prime}$ and $\lambda_{112}^{\prime}$ couplings should have similar magnitudes. This must be made consistent with the constraint $\left(L_{1} Q_{2} \bar{D}_{1}\right) \cdot\left(L_{1} Q_{1} \bar{D}_{2}\right) \leq 4 \cdot 10^{-9}$, which arises from bounds on $\Delta m_{K}$. In the present context, this constraint indicates that the relevant charge $|7+w|$ has to be large.

The problem gets more complicated by taking into account mixing effects, which for left-right symmetric models have been studied in detail in [42], in combination with bounds 

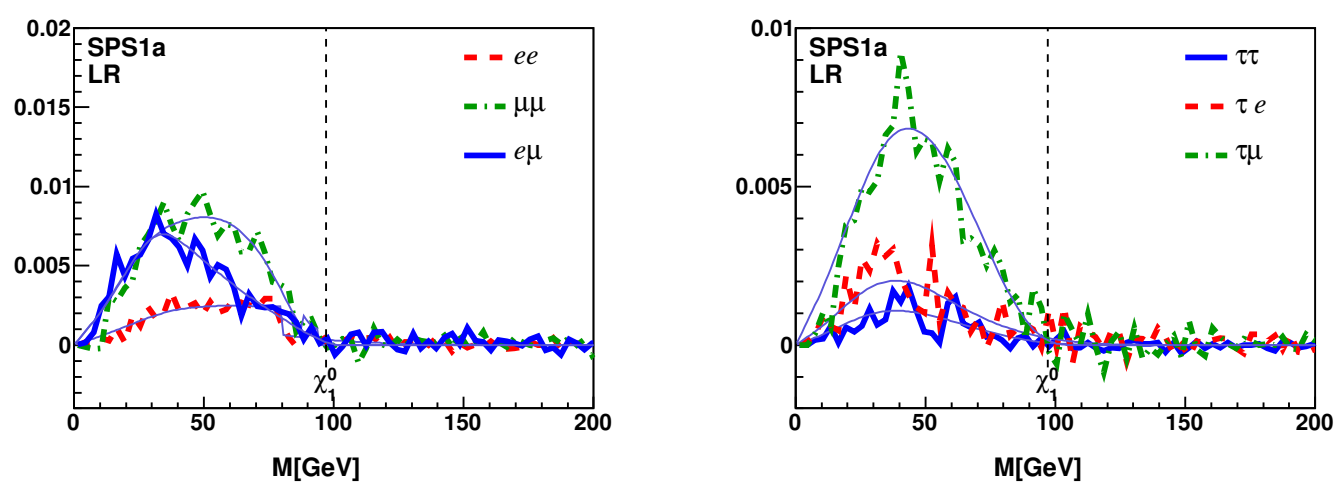

Figure 16. The same as figure 5 for the Left-Right symmetric scenario defined by (6.4) at SPS1a.

on both individual couplings as well as on products. Summarising, it turned out that the strong correlations were leading to a suppression of all couplings, and even the 333 flavour, in order to be fully safe, had to be smaller than 0.006. Within this framework, therefore, single superparticle productions are suppressed and the best signal would be pair productions followed by $R$-violating decays (which favour the neutralino search channel even further). Finally, quantum corrections, expressed through renormalisation group effects, would only mildly affect the relative hierarchies of R-violating couplings (although they would modify their absolute values between the GUT and the low energy scale) [18].

For the $\Delta L \neq 0$ operators, we may then study the correlated operators:

$$
L L \bar{E}_{\text {eff }}+L Q \bar{D}_{\text {eff }}
$$

with

$$
\begin{aligned}
L L \bar{E}_{\text {eff }} & =\bar{\epsilon} L_{2} L_{3} \bar{E}_{3}+\bar{\epsilon}^{2} L_{1} L_{2} \bar{E}_{2}+\mathcal{O}\left(\bar{\epsilon}^{3}\right), \\
L Q \bar{D}_{\text {eff }} & =L_{3} Q_{3} \bar{D}_{3}+\bar{\epsilon}\left(L_{2} Q_{3} \bar{D}_{3}+L_{3} Q_{2} \bar{D}_{3}+L_{3} Q_{3} \bar{D}_{2}\right)+\mathcal{O}\left(\bar{\epsilon}^{2}\right),
\end{aligned}
$$

Due to the larger decay widths associated with the $L L \bar{E}$ operators as compared to the $L Q \bar{D}$ operators as well as the lack of a clear signal associated with the $Q_{3}$ factor in the dominant operator; the only thing we can see is the $L_{2} L_{3} \bar{E}_{3}$ operator and possibly a hint of the $L_{1} L_{2} \bar{E}_{2}$ operator. This is illustrated in figure 16 where the leptonic invariant mass distributions are plotted. The data is consistent with a dominant contribution from a $\mu \tau$ decay channel giving the $\mu \tau_{\text {jet }}$ distribution, together with contributions of type (B.11) to the $\mu \mu$ and $e \mu$ distributions. Since the $\mu \mu$ and $e \mu$ distributions have kinematical cutoffs of the same scale as the $\mu \tau_{\text {jet }}$ distribution, we need an additional contribution of type (B.2) to those distributions, consistent with a subdominant $L_{1} L_{2} \bar{E}_{2}$ operator.

Due to the dominant $L Q \bar{D}$ operator, a larger fraction of the leptons in the final state will come from the upper decay chain rather than the neutralino decay, as compared to the pure $L L \bar{E}$ case, and therefore the numerical observables of section 3.6 are too dependent on SPS point and not very useful. This contamination also causes the invariant mass distributions to be much less smooth and therefore more difficult to interpret. 


\begin{tabular}{|c|c|c|c|c|c|c|}
\hline$i j k$ & 121,131 & 231 & 122,132 & 232 & 123,133 & 233 \\
\hline $\mathrm{U}(1)$ & $4-w$ & $3-w$ & $3-w$ & $2-w$ & $1-w$ & $-w$ \\
\hline
\end{tabular}

Table 12. $L L \bar{E}$ charges in $\mathrm{SU}(5)$ enhanced by a U(1) flavour symmetry.

If we now look at the $\bar{U} \bar{D} \bar{D}$ operators, we see that even though, within this model, the largest coupling is $\bar{U}_{3} \bar{D}_{2} \bar{D}_{3}$, the phase-space suppression caused by the top quark in the final state makes the dominant channels the ones with three quark jets, even at SPS6 where the neutralino is sufficiently heavy to decay to a top.

\subsection{2 $\mathrm{SU}(5)$}

Another interesting possibility is that the family symmetry commutes with an SU(5) GUT, where the SM fermions are assigned as follows to the representations of the group:

$$
\begin{aligned}
Q_{\left(q, u^{c}, e^{c}\right)_{i}} & =Q_{i}^{10} \\
Q_{\left(l, d^{c}\right)_{i}} & =Q_{i}^{\overline{5}} \\
Q_{\left(\nu_{R}\right)_{i}} & =Q_{i}^{\nu_{R}}
\end{aligned}
$$

From the above it immediately follows that:

1. The up-quark mass matrix is symmetric (both left- and right-handed up quarks are in the 10, and thus have the same flavour charge).

2. the charged lepton mass matrix is the transpose of the down quark mass matrix.

In this case, a viable choice of charges obeying the restrictions of the symmetry (see e.g., [69]), is:

$$
\begin{aligned}
& Q_{1,2,3}=\bar{E}_{1,2,3}=3,2,0 \\
& \bar{D}_{1,2,3}=L_{1,2,3}=1,0,0
\end{aligned}
$$

leading to matrices that, apart from other features, lead to a maximal 2-3 lepton mixing, viz.

$$
M^{\text {up }} \sim\left(\begin{array}{ccc}
\bar{\epsilon}^{6} & \bar{\epsilon}^{5} & \bar{\epsilon}^{3} \\
\bar{\epsilon}^{5} & \bar{\epsilon}^{4} & \bar{\epsilon}^{2} \\
\bar{\epsilon}^{3} & \bar{\epsilon}^{2} & 1
\end{array}\right), \quad M^{\text {down }} \sim\left(\begin{array}{ccc}
\bar{\epsilon}^{4} & \bar{\epsilon}^{3} & \bar{\epsilon}^{3} \\
\bar{\epsilon}^{3} & \bar{\epsilon}^{2} & \bar{\epsilon}^{2} \\
\bar{\epsilon} & 1 & 1
\end{array}\right), \quad M^{\ell} \sim\left(\begin{array}{ccc}
\bar{\epsilon}^{4} & \bar{\epsilon}^{3} & \bar{\epsilon} \\
\bar{\epsilon}^{3} & \bar{\epsilon}^{2} & 1 \\
\bar{\epsilon}^{3} & \bar{\epsilon}^{2} & 1
\end{array}\right),
$$

where $\bar{\epsilon} \approx 0.2$.

Let us first look at the implications for the $L L \bar{E}$ operators. Since the charges of $L_{2,3}$ are the same, couplings such as $L_{i} L_{2} \bar{E}_{k}$ and $L_{i} L_{3} \bar{E}_{k}$ would be expected to be of similar magnitude. In short, the U(1) assignments of eq. (6.7) lead to operator charges as listed in table 12 .

Similarly for $L Q \bar{D}$, where now we have the connection $\lambda_{i j k}^{\prime}=\lambda_{i j k}$ arising directly from the way we accommodate the fields in the GUT representation. For the $L Q \bar{D}$ operators we also note the following: 


\begin{tabular}{|c|c|c|c|c|c|c|}
\hline$i j k^{\prime}$ & 111 & 112,113 & 121 & 122,123 & 131 & 132,133 \\
\hline $\mathrm{U}(1)$ & $5-w$ & $4-w$ & $4-w$ & $3-w$ & $2-w$ & $1-w$ \\
\hline$i j k^{\prime}$ & 211,311 & $212,213,312$ & 221,321 & $222,223,322,323$ & 231 & $232,233,332,333$ \\
\hline $\mathrm{U}(1)$ & $4-w$ & $3-w$ & $3-w$ & $2-w$ & $1-w$ & $-w$ \\
\hline
\end{tabular}

Table 13. $L Q \bar{D}$ charges in $\mathrm{SU}(5)$ enhanced by a $\mathrm{U}(1)$ flavour symmetry.

\begin{tabular}{|c|c|c|c|c|c|c|}
\hline$i j k^{\prime \prime}$ & 112,113 & 123 & 212,213 & 223 & 312,313 & 323 \\
\hline $\mathrm{U}(1)$ & $4-w$ & $3-w$ & $3-w$ & $2-w$ & $1-w$ & $-w$ \\
\hline
\end{tabular}

Table 14. $\bar{U} \bar{D} \bar{D}$ charges in $\mathrm{SU}(5)$ enhanced by a U(1) flavour symmetry.
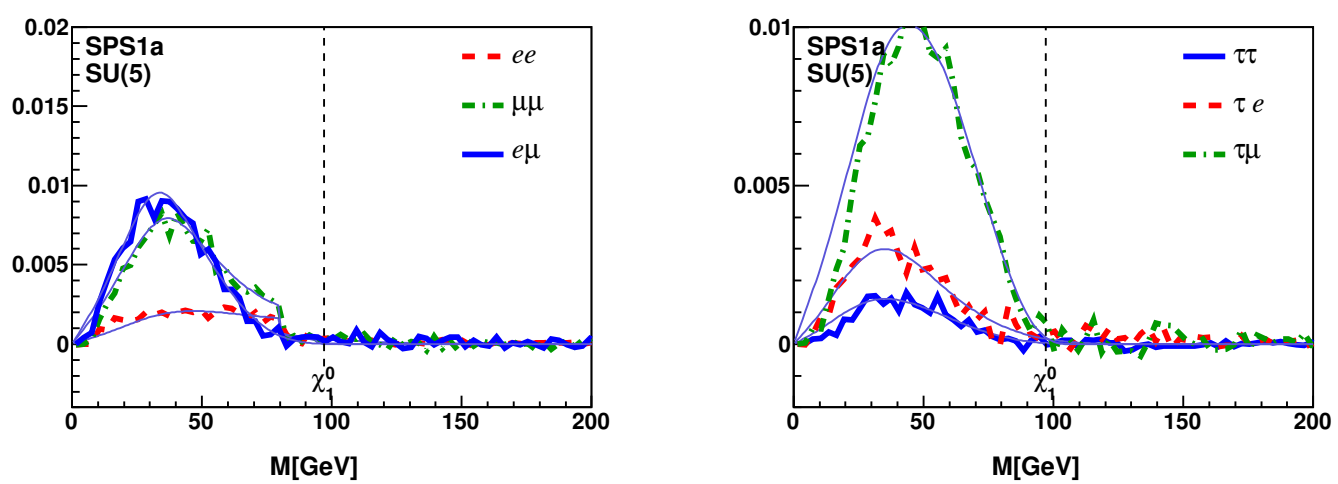

Figure 17. The same as figure 5 for the SU(5) scenario defined by tables 12 and 13 at SPS1a.

- Since the U(1) charges of $L_{2,3}$ are the same, the respective operators are linked, reducing the number of independent couplings.

- Since the U(1) charges of $\bar{D}_{2,3}$ are the same, the respective operators are linked, reducing the number of independent couplings.

This leaves us with the results of table 13. Finally, for the baryon-number violating operators, we find the results given in table 14 .

As for the $L$-violating part, for $w=0$, the $L_{2} L_{3} \bar{E}_{3}$ coupling is as large as the largest of the $L Q \bar{D}$ couplings and therefore the prospects of accurately identifying this coupling are improved. Moreover, the leading subdominant $L L \bar{E}$ operators, namely $L_{1} L_{3} \bar{E}_{3}$ and $L_{1} L_{2} \bar{E}_{3}$, also carry a lot of tau flavour. This ensures an even larger dominance of the $L_{2} L_{3} \bar{E}_{3}$ operator after cuts are applied (in the Left-Right symmetric case the cuts partially compensate for the difference in width between the $L_{2} L_{3} \bar{E}_{3}$ and the $L_{1} L_{2} \bar{E}_{2}$ operator). All in all, the prospects of identifying the $L_{2} L_{3} \bar{E}_{3}$ operator are good but it does not appear possible to extract any of the subdominant couplings.

This can be seen in the invariant mass distributions shown in figure 17 where all distributions look much like we expect for a pure $L_{2} L_{3} \bar{E}_{3}$ operator. Note that all the light lepton distributions of the left panel of figure 17 vanish between $\approx 80 \mathrm{GeV}$ and the 
kinematical end-point of the $\mu \tau_{\text {jet }}$ distribution $\left(\approx M_{\chi_{1}^{0}}\right)$ and we can therefore exclude any significant neutralino decay to light leptons.

Note that this strong statement is linked to the simplifying assumption that like for the Yukawa couplings, R-violating couplings of the third generation are in general larger. If this assumption is dropped, the picture would change. For instance, for the $L L \bar{E}$ operators:

- $w=1$ would imply a simultaneous dominance of the $L_{1} L_{2} \bar{E}_{3}$ and $L_{1} L_{3} \bar{E}_{3}$ couplings,

- $w=2$ leads to a dominant $L_{2} L_{3} \bar{E}_{2}$ coupling, and

- $w=3$ results in a simultaneous dominance of the $L_{2} L_{3} \bar{E}_{1}, L_{1} L_{2} \bar{E}_{2}$ and $L_{1} L_{3} \bar{E}_{2}$ couplings.

A similar situation (but for different respective flavours) would occur for the rest of the operators.

For the baryon-violating case, $w=0$ leads to a stronger dominance of the $\bar{U}_{3} \bar{D}_{j} \bar{D}_{k}$ operators as compared to the Left-Right symmetric case. Consequently, the decay to top plus 3 jets will dominate if the neutralino is heavy enough, e.g. in SPS6.

\section{Summary}

Within a supersymmetric theory with trilinear R-parity violation and a neutralino LSP, ${ }^{10}$ one would expect the usual pair production of squarks and gluinos, followed by cascade decays down to the neutralino, to be the dominant scenario at the LHC. The novel feature introduced by the R-parity violation would then be the three-body decay of the neutralino.

Within such a model, the neutralino can decay through any of the 45 trilinear R-parity violating operators and therefore this channel allows us to study all those operators and their internal hierarchies simultaneously. On account of the typically large number of detectable particles (especially leptons) produced in such neutralino decays, the inferences drawn from such a study are not as dependent on the underlying SUSY scenario as R-parity conserving SUSY scenarios can be.

In general, bilinear and trilinear terms are generated in different ways (for instance an underlying string theory would favour trilinear couplings; on the other hand, flavour symmetries could in principle favour either possibility). See, however, ref. [78]. The decay modes $\tilde{\chi}_{1}^{0} \rightarrow W^{ \pm} \ell^{\mp}$ and $\tilde{\chi}_{1}^{0} \rightarrow Z \nu$ exist in bilinear schemes, leading to interesting signatures $[79,80]$. For these terms to dominate, one would require non-negligible neutrino-neutralino mixing.

We have shown that the prospects of identifying trilinear operators of the $L L \bar{E}$ type are rather good, mostly due to the abundance of invariant mass distributions one can explore. While one can, in principle, also identify such operators from pure counting measures, the corresponding conclusions would not only be more sensitive to backgrounds, but also to potential competing operators, as well as to the SUSY model. In addition to a significant

\footnotetext{
${ }^{10}$ The lightest neutralino could even be the NLSP if, for example, we invoke a gravitino LSP for dark matter; such an assumption does not affect this study.
} 
reduction in such dependences, the invariant mass distributions are also promising for measuring the neutralino mass; this has been shown to be feasible thanks to the knowledge of the theoretically expected distributions, despite the lack of clear peaks or edges.

As compared to $L L \bar{E}$ operators, $L Q \bar{D}$ operators have both advantages and disadvantages. One disadvantage is that background in the form of $t \bar{t}$ events is more challenging, but the large lepton multiplicity is still sufficient to get a clean event sample. Operators that lead to charged leptons and jets also have the advantage of producing peaks in the relevant invariant mass distributions, which not only allow good determination of the operator, but also accurate measurements of the neutralino mass. Particularly promising are the operators leading to $b$-quarks in the decay since, in this case, $b$-tagging can also be used to extract the signal.

With a tau as the lepton of the operator, things are not equally promising, due to the loss of energy to neutrinos in the tau decay. However, if the tau is accompanied by a $b$-quark, then $b$ and tau tagging together might provide the information we need. For the case with a tau and two light jets, and for $L Q_{3} \bar{D}$ operators (which only gives neutrino and two jets) we do not have an obvious method of identifying the operator.

If more than one lepton number violating operators are large at the same time, we have shown that the prospects of identifying them are good (in most cases, the signals we are looking for appear in different channels for different operators and these signals are mostly independent of each other). It is also worth mentioning that if both $L L \bar{E}$ and $L Q \bar{D}$ operators are large at the same time we expect to see only the $L L \bar{E}$ operator since it gets a larger branching fraction and is easier to detect.

From flavour models one may expect large R-violating hierarchies, similar to those of the Yukawa couplings that generate fermion masses. Fermion mass terms are dominated by the heavier flavours. If this feature persists for the R-violating couplings as well, it would make the identification of the $L Q \bar{D}$ operators very difficult since the dominant ones will either contain tau flavour or a $Q_{3}$ operator, with either case implying a low chance of identification. In addition, accompanying $L L \bar{E}$ operators have the advantage of larger branching fraction as well as easier detection. The conclusion is that in this subclass of flavour models we would expect to see dominant $L L \bar{E}$ operators with heavy flavours, i.e. lots of tau flavour.

Finally we have looked at the case where a heavy neutralino $\left(M_{\chi_{1}^{0}}>M_{\text {top }}\right)$ decays via a $\bar{U}_{3} \bar{D}_{j} \bar{D}_{k}$ operator to a top quark and two jets, and have shown that this scenario can be identified. This is important for flavour physics since in many models the R-parity violating couplings with heavy flavours will be the dominant ones.

\section{Acknowledgments}

We thank the NORDITA program "TeV scale physics and dark matter", for hospitality while this work was initiated. SL thanks the CERN Theory Division, for kind hospitality. The research of PO has been supported by the Research Council of Norway. 


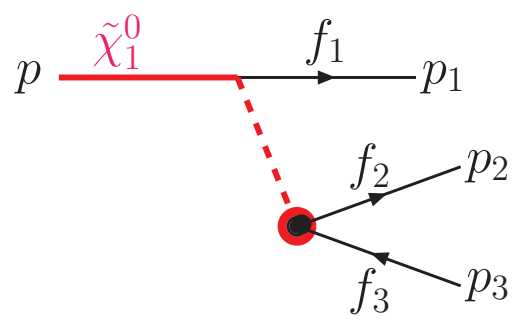

Figure 18. Kinematics of three-body neutralino decay.

\section{A Energy spectra from three-body decays}

For any decay into a final state comprising more than two particles, the energy distribution of a given final state particle is determined not only by kinematics, but has a dependence on the dynamics as well. In particular, let us consider the decay of a neutralino to three fermions, namely

$$
\tilde{\chi}_{1}^{0} \rightarrow f_{1} f_{2} f_{3} .
$$

There are three contributions, characterized by the identity of the sfermion (corresponding to the fermion coupling to the neutralino). Adopting the notation of figure 18, the corresponding matrix element will be of the form

$$
\mathcal{M} \sim\left[\bar{u}\left(p_{1}\right) u(p)\right]\left[\bar{u}\left(p_{2}\right) v\left(p_{3}\right)\right] .
$$

Here, we disregard the energy dependence of the propagator (valid for a heavy sfermion) and the pseudoscalar part of the coupling, since its presence will not modify any differential decay rate. Then,

$$
\sum_{\text {spins }} \mathcal{M}^{\dagger} \mathcal{M} \propto\left(p \cdot p_{1}\right)\left(p_{2} \cdot p_{3}\right)
$$

In the decay, two of the final-state particles will be charged, and potentially observable. For a particular final-state particle, the energy distribution depends on whether it comes from the upper or lower vertex in figure 18. With the notations $p=\{M, \mathbf{0}\}, p_{i}=\left\{E_{i}, \boldsymbol{p}_{i}\right\}$, and $\epsilon_{i}=E_{i} / E_{i}^{\max } \equiv 2 E_{i} / M$, we find

$$
\frac{1}{\Gamma} \frac{d \Gamma}{d \epsilon_{i}}= \begin{cases}12 \epsilon_{i}^{2}\left(1-\epsilon_{i}\right) & i=1, \\ 2 \epsilon_{i}^{2}\left(3-2 \epsilon_{i}\right) & i=2,3 .\end{cases}
$$

For $i=1$, the distribution vanishes at the upper end due to angular momentum conservation. For comparison, if the distribution had been determined by kinematics alone, it would have read

$$
\frac{1}{\Gamma} \frac{d \Gamma}{d \epsilon_{i}}=2 \epsilon_{i}
$$

To see the implications of this, consider the operator $L_{1} L_{2} \bar{E}_{3}$. The last factor represents a coupling to a tau or a stau (both charged), whereas the first two (by SU(2) invariance) represent couplings to a charged field and a neutral one, one of which will be of the first family, whereas the other will be of the second family. We thus have two distinct decay channels:

$$
\tilde{\chi}_{1}^{0} \rightarrow \tau^{+} e^{-} \nu_{\mu}, \quad \tilde{\chi}_{1}^{0} \rightarrow \tau^{+} \mu^{-} \nu_{e},
$$




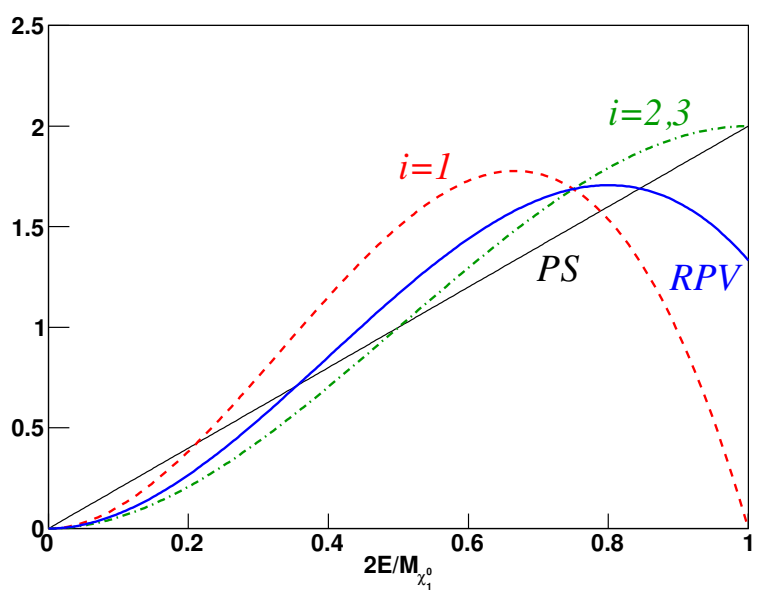

Figure 19. Energy distributions for the decay products in a three body decay, eq. (A.4). The straight black line shows the distribution achieved when only phase space (PS) is taken into account. The average, eq. (A.7), is denoted 'RPV'.

and charge conjugates. Each of these channels is governed by three diagrams of the type shown in figure 18. Assuming that all the sfermions have comparable masses, and neglecting interferences, the energy distribution for each of the decay products would be averaging the distributions (A.4) over all three values of $i$, resulting in

$$
\frac{1}{\Gamma} \frac{d \Gamma}{d \epsilon}=\epsilon^{2}\left(8-\frac{20}{3} \epsilon\right)
$$

The above assumptions are not entirely justified, e.g. we do expect differences in sparticle masses to introduce differences between the three diagrams due to propagator effects and we have no reason to ignore interference altogether. However, eq. (A.7) should still be a good approximation and the assumptions involved provide a useful formula.

The distributions (A.4), (A.7) as well as (A.5) are shown in figure 19.

\section{B Theoretical invariant mass distributions}

In order to calculate the theoretically expected invariant mass distributions [72-77] from the leptonic neutralino decays, we start with the energy distribution of the decay products, as given by eq. (A.7).

Let us now denote the three decay products $a, b$ and $c$, with corresponding fourmomenta $p_{a}, p_{b}$ and $p_{c}$. The invariant mass $M_{a b}$ of particles $a$ and $b$ is given by

$$
M_{a b}^{2}=\left(p_{a}+p_{b}\right)^{2}=M^{2}-2 M E_{c}+m_{c}^{2}
$$

where $E_{c}$ denotes the energy in the neutralino rest frame and $M$ is the neutralino mass. Assuming that particle $c$ is massless and follows the distribution given by eq. (A.7) we get:

$$
f_{M_{a b}}\left(M_{a b}\right)=\frac{8 M_{a b}}{3 M^{8}}\left(M^{2}-M_{a b}^{2}\right)^{2}\left(M^{2}+5 M_{a b}^{2}\right) .
$$



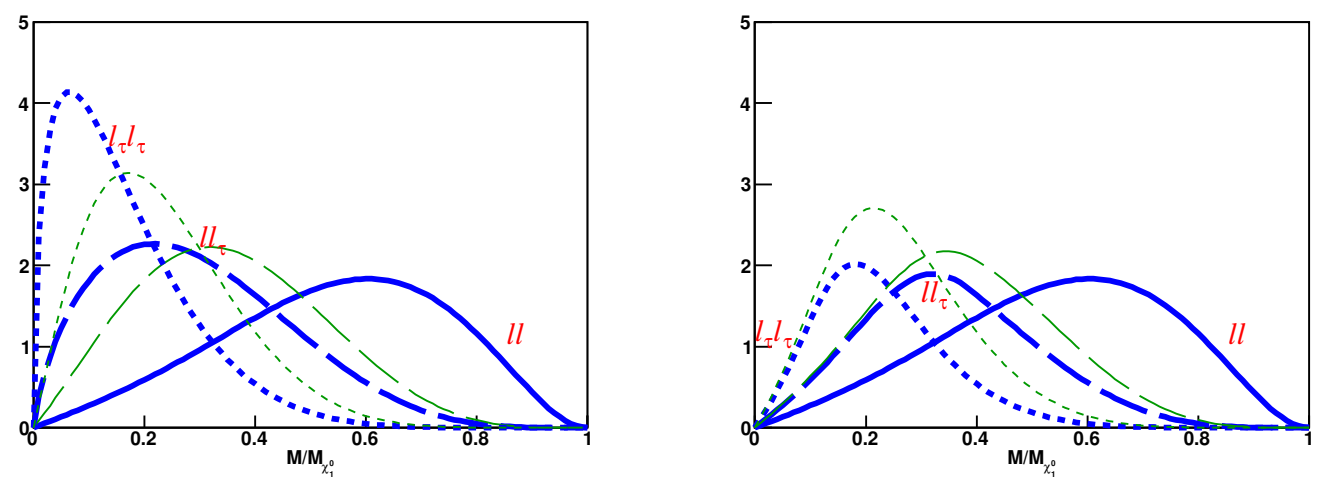

Figure 20. Theoretical invariant di-lepton mass distributions for massless particles from $\tilde{\chi}_{1}^{0}$ decay. Left: No cuts. The $\ell \ell$ curve is given by eq. (B.2). Right: Cuts as indicated by eq. (B.11). Solid curves: directly produced leptons; long-dashed curves: one lepton comes from a decaying tau; short-dashed curves: both leptons come from decaying taus. Blue heavy curves do not take tau polarization into account while the thin green curves do. The labels refer to the heavy curves (blue).

This distribution is shown in figure 20 (solid curves).

If one of the charged particles is a $\tau$, we get a contribution to the di-lepton invariant mass only if the $\tau$ decays leptonically. To calculate the distribution from this, we need to estimate a distribution for the fraction of the energy of the tau that is transferred to the charged lepton.

To do this, we assume that the tau decays into three massless particles (one charged lepton and two neutrinos). The kinematics of a tau decay will be the same as discussed in appendix A for a neutralino decay. However, with a $V-A$ coupling, $i=1$ in eq. (A.4) represents the antiparticle among the decay products. In the rest frame of the tau, the charged lepton will then follow the distribution for $i=2,3$ with $M=M_{\tau}$.

The next step is to perform a Lorentz boost to the rest frame of the neutralino. If we denote the momentum four-vector of the charged lepton in the rest frame of the tau, $\left(E_{l}, \boldsymbol{P}_{l}\right)$ and that of the tau in the rest frame of the neutralino, $(E, \boldsymbol{P})$, we get the energy of the lepton in the rest frame of the neutralino as:

$$
\frac{E_{l} E}{M_{\tau}}+\frac{\boldsymbol{P}_{l} \cdot \boldsymbol{P}}{M_{\tau}}
$$

Since we assume that the lepton is massless, i.e., $E_{l}=\left|\boldsymbol{P}_{l}\right|$, and that the lepton carries a fraction $x$ of $M_{\tau} / 2$ in the rest frame of the tau, i.e. $E_{l}=x M_{\tau} / 2$, eq. (B.3) can be written:

$$
\frac{x}{2}(E+|\boldsymbol{P}| \cos \theta)
$$

where $\theta$ is the angle between $\boldsymbol{P}$ and $\boldsymbol{P}_{l}$. Let us now take the limit of a massless tau, i.e., $E=|\boldsymbol{P}|$, then we get:

$$
\frac{x E}{2}(1+\cos \theta)
$$


Assuming that the direction of the lepton is chosen isotropically ${ }^{11}$ in the rest frame of the tau, implies that the distribution of $\cos \theta$ is flat. From that we can calculate the distribution of $y=1+\cos \theta$ as:

$$
f_{y}(y)=\frac{1}{2},
$$

where $y \in[0,2]$. We are interested in the fractional lepton energy with respect to that of the tau, in the $\tilde{\chi}_{1}^{0}$ rest frame, i.e., the product $z=\frac{x}{2} y$ where $x$ by construction follows the distribution (A.4) with $i=2,3$. The distribution $f_{z}(z)$ is now given by:

$$
f_{z}(z)=\frac{5-9 z^{2}+4 z^{3}}{3}
$$

With all decay products from the neutralino massless, we can simplify the invariant mass formula:

$$
M_{a b}^{2}=\left(p_{a}+p_{b}\right)^{2}=2 E_{a} E_{b}(1-\cos \phi),
$$

where $\phi$ is the angle between $\boldsymbol{P}_{a}$ and $\boldsymbol{P}_{b}$. If we then assume that particle $b$ is a tau and decays to a charged lepton $d$, the resulting invariant mass $M_{a d}$ is given by:

$$
M_{a d}=M_{a b} q
$$

where $q=\sqrt{z}$ and has a distribution $f_{q}(q)=\frac{2 q}{3}\left(5-9 q^{4}+4 q^{6}\right) . M_{a d}$ then has a distribution:

$$
f_{M_{a d}}\left(M_{a d}\right)=\int_{M_{a d} / M}^{1} \frac{f_{q}(q) f_{M_{a b}}\left(M_{a d} / q\right)}{q} d q .
$$

We see from eq. (B.7) that there are many low-energy leptons coming from the tau decay. These low-energy leptons will, however, not pass the $p_{T}$ cuts we impose and therefore we need to take the cuts into account when calculating the distribution (this is not required for the direct decay to leptons since the amount of low energy leptons there is small). The simplest thing to do to include some cuts, is to constrain the lower limit in the integral of eq. (B.10):

$$
f_{M_{a d}}\left(M_{a d}\right)=\int_{\max \left(M_{a d} / M, 0.4\right)}^{1} \frac{f_{q}(q) f_{M_{a b}}\left(M_{a d} / q\right)}{q} d q .
$$

The introduction of this cut does not preserve the normalization of the distribution; up to this point all distributions have been normalized to unity as one would expect for probability distributions. However, this is no longer true for eq. (B.11), moreover, the new normalization will depend on the distribution to which this kind of cut is applied, due to the larger impact on the lower end of the distribution.

Since we are more concerned about the shape rather than the exact values from the distributions, there is no need to normalize eq. (B.11). Note also that introducing the cuts this way is simple and gives a reasonably good result, but it is very ad hoc, the specific value 0.4 is adopted for no other reason than the fact that it gives a good approximation to the Monte Carlo results.

\footnotetext{
${ }^{11}$ Since the tau will in general be left-handed, this is not a very accurate assumption. For a discussion of the effects of tau polarization, see below.
} 

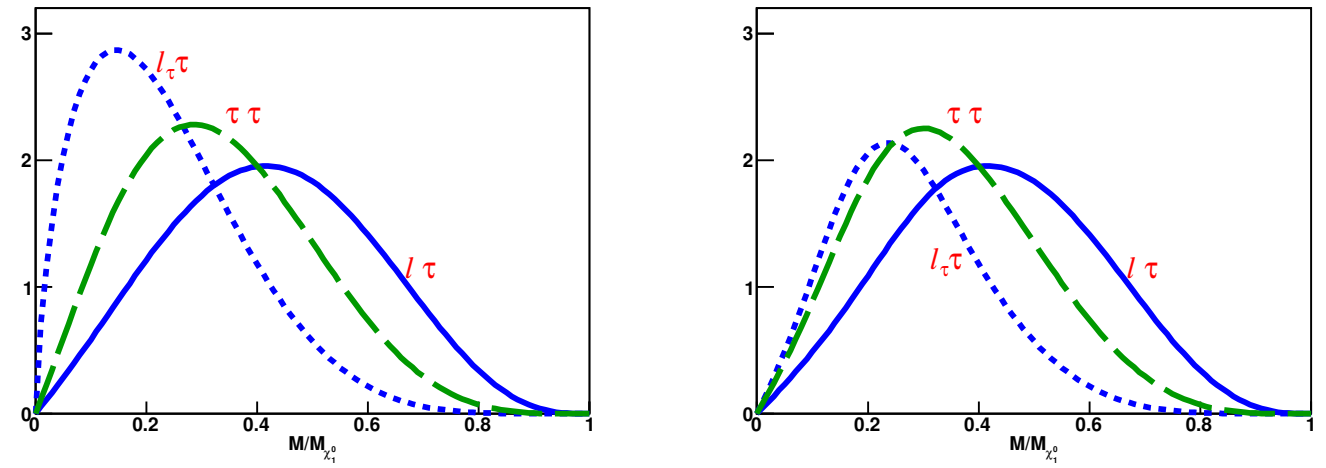

Figure 21. Effects of cuts on invariant mass distributions involving taus. Left: No cuts. Right: Cuts as indicated by eq. (B.11). Long-dashed (green) curve: two tau jets are combined; solid (blue): one directly produced lepton combined with a tau jet; short-dashed (blue): a tau together with a lepton from a tau decay.
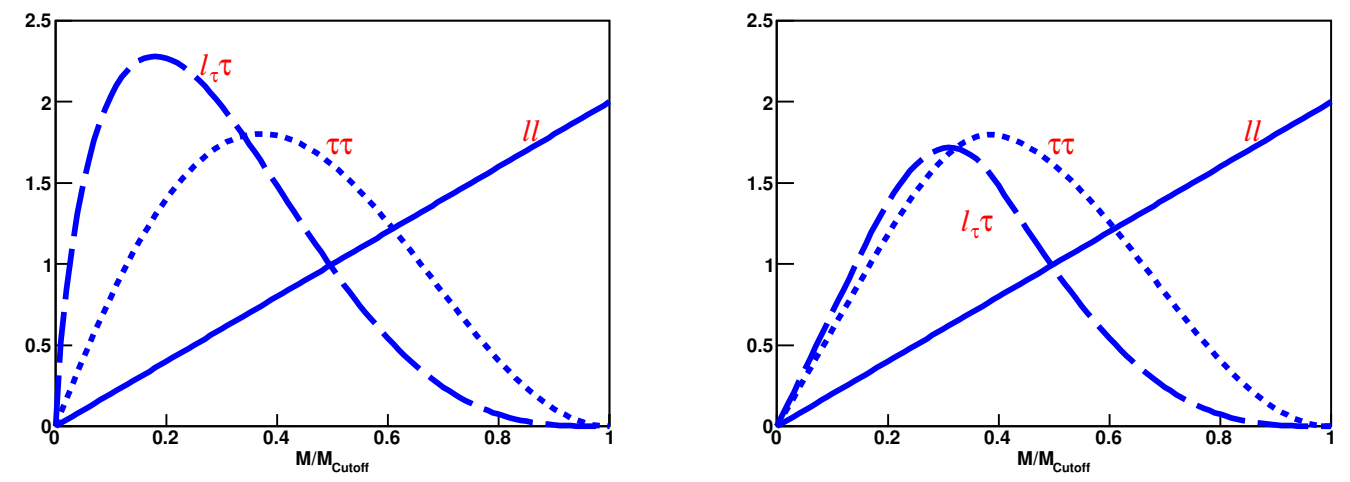

Figure 22. Invariant mass distributions expected from the higher RPC chain. Left: No cuts. Right: Cuts as indicated by eq. (B.11). Solid: the familiar triangular di-lepton distribution; shortdashed: the di-tau-jet invariant mass; long-dashed: lepton-tau-jet mass distribution, expected when one of the taus decays leptonically.

We can also extend the analysis to the case when both leptons come from decaying taus by multiplying $M_{a d}$ with $q=\sqrt{z}$ once again. The result of this is shown as the long-dashed curves of figure 20 and the distributions of eq. (B.10) and eq. (B.11) are shown as the short-dashed curves.

So far we have ignored any effects of tau polarization. However, since the neutralino will in general decay to left-handed taus, the spin of the tau will affect the mass distribution. More precisely, we get an extra factor $\frac{1}{2}(1+\cos \theta)$ which amounts to replacing eq. (B.6) with $f_{y}(y)=\frac{y}{2}$. The result of this can be seen in the thin green curves of figure 20. The tau polarization pushes the distributions to higher energy but the effect is smaller than the uncertainties regarding the effects of the cuts. Given that PYTHIA also does not take polarization into account, we use the distributions without polarization effects for the comparison with Monte Carlo. 
In the right panel of figure 20 we see the result of simulating the cuts with a constraint on the integral as done in eq. (B.11). As mentioned before, this suppresses the lower end of the distributions and changes the normalizations. We can see that the change in normalization is different without (blue curves) and with tau polarization effects (green curves). Including tau polarization effects, the distributions are pushed towards higher masses and are therefore less affected by the cuts.

If the tau instead decays hadronically, we need to estimate the energy of the resulting tau-jet $\left(\tau_{\text {jet }}\right)$. The simplest thing to do is to assume a three-body decay of the tau where the neutrino gets a fraction $c$ of the tau mass and the rest of the energy goes into the $\tau_{\text {jet }}$. The distribution of $c$ will then follow (A.4) for $i=2,3$. We get the invariant mass of a $\tau_{\text {jet }}$ combined with a lepton, $M_{\tau_{\mathrm{jet}} l}$ as:

$$
M_{\tau_{\mathrm{jet}} l}=M_{a b} \sqrt{1-c}
$$

where $M_{a b}$ and $c$ are as defined above. In line with the above procedure we introduce the cuts by requiring $\sqrt{1-c}>0.4$.

This procedure can be repeated to produce the distribution of two $\tau_{\text {jet's }}$ as well as lepton- $\tau_{\text {jet }}$ distributions where the lepton comes from the decay of a tau. The result of this is shown in figure 21.

For comparison, in figure 22 we show the expected signals from the RPC chain. One clearly sees the similarities between the RPV and RPC signals in channels including $\tau_{\text {jet }}$ 's. This makes the study of these channels much more difficult.

Open Access. This article is distributed under the terms of the Creative Commons Attribution Noncommercial License which permits any noncommercial use, distribution, and reproduction in any medium, provided the original author(s) and source are credited.

\section{References}

[1] P. Fayet, Spontaneously broken supersymmetric theories of weak, electromagnetic and strong interactions, Phys. Lett. B 69 (1977) 489 [SPIRES].

[2] G.R. Farrar and P. Fayet, Phenomenology of the production, decay and detection of new hadronic states associated with supersymmetry, Phys. Lett. B 76 (1978) 575 [SPIRES].

[3] L.E. Ibáñez and G.G. Ross, Discrete gauge symmetry anomalies, Phys. Lett. B 260 (1991) 291 [SPIRES].

[4] L.E. Ibáñez and G.G. Ross, Discrete gauge symmetries and the origin of baryon and lepton number conservation in supersymmetric versions of the standard model, Nucl. Phys. B 368 (1992) 3 [SPIRES].

[5] S. Lola and G.G. Ross, Baryon and lepton number (non)conservation in supersymmetric theories, Phys. Lett. B 314 (1993) 336 [SPIRES].

[6] A.Y. Smirnov and F. Vissani, Upper bound on all products of R-parity violating couplings $\lambda^{\prime}$ and $\lambda^{\prime \prime}$ from proton decay, Phys. Lett. B 380 (1996) 317 [hep-ph/9601387] [SPIRES].

[7] J.L. Goity and M. Sher, Bounds on $\Delta B=1$ couplings in the supersymmetric standard model, Phys. Lett. B 346 (1995) 69 [Erratum ibid. B 385 (1996) 500] [hep-ph/9412208] [SPIRES]. 
[8] B. Brahmachari and P. Roy, Constraints on baryon nonconserving Yukawa couplings in a supersymmetric theory, Phys. Rev. D 50 (1994) R39 [Erratum ibid. D 51 (1995) 3974] [hep-ph/9403350] [SPIRES].

[9] D. Chakraverty and D. Choudhury, $b \rightarrow s \gamma$ confronts $B$ violating scalar couplings: R-parity violating supersymmetry or diquark, Phys. Rev. D 63 (2001) 075009 [hep-ph/0008165] [SPIRES].

[10] D. Chakraverty and D. Choudhury, B physics constraints on baryon number violating couplings: grand unification or R-parity violation, Phys. Rev. D 63 (2001) 112002 [hep-ph/0012309] [SPIRES].

[11] G. Bhattacharyya and D. Choudhury, $D$ and $\tau$ decays: placing new bounds on R-parity violating supersymmetric coupling, Mod. Phys. Lett. A 10 (1995) 1699 [hep-ph/9503263] [SPIRES].

[12] G. Bhattacharyya, J.R. Ellis and K. Sridhar, New LEP constraints on some supersymmetric Yukawa interactions that violate R-parity, Mod. Phys. Lett. A 10 (1995) 1583 [hep-ph/9503264] [SPIRES].

[13] G. Bhattacharyya, D. Choudhury and K. Sridhar, New LEP bounds on B violating scalar couplings: R-parity violating supersymmetry or diquarks, Phys. Lett. B 355 (1995) 193 [hep-ph/9504314] [SPIRES].

[14] M. Hirsch, H.V. Klapdor-Kleingrothaus and S.G. Kovalenko, New constraints on R-parity broken supersymmetry from neutrinoless double beta decay, Phys. Rev. Lett. 75 (1995) 17 [SPIRES].

[15] G. Bhattacharyya, A brief review of R-parity-violating couplings, hep-ph/9709395 [SPIRES].

[16] G. Bhattacharyya, R-parity-violating supersymmetric Yukawa couplings: a mini-review, Nucl. Phys. Proc. Suppl. 52A (1997) 83 [hep-ph/9608415] [SPIRES].

[17] H.K. Dreiner, An introduction to explicit R-parity violation, hep-ph/9707435 [SPIRES].

[18] B.C. Allanach, A. Dedes and H.K. Dreiner, Bounds on R-parity violating couplings at the weak scale and at the GUT scale, Phys. Rev. D 60 (1999) 075014 [hep-ph/9906209] [SPIRES].

[19] For an introduction to the phenomenology of the MSSM see H.P. Nilles, Supersymmetry, supergravity and particle physics, Phys. Rept. 110 (1984) 1 [SPIRES].

[20] H.E. Haber and G.L. Kane, The search for supersymmetry: probing physics beyond the standard model, Phys. Rept. 117 (1985) 75 [SPIRES].

[21] For some of the earliest references on the phenomenology of R-violating supersymmetry see F. Zwirner, Observable $\Delta B=2$ transitions without nucleon decay in a minimal supersymmetric extension of the standard model, Phys. Lett. B 132 (1983) 103 [SPIRES].

[22] J.R. Ellis, G. Gelmini, C. Jarlskog, G.G. Ross and J.W.F. Valle, Phenomenology of supersymmetry with broken R-parity, Phys. Lett. B 150 (1985) 142 [SPIRES].

[23] G.G. Ross and J.W.F. Valle, Supersymmetric models without R-parity, Phys. Lett. B 151 (1985) 375 [SPIRES].

[24] S. Dimopoulos and L.J. Hall, Lepton and baryon number violating collider signatures from supersymmetry, Phys. Lett. B 207 (1988) 210 [SPIRES].

[25] L.J. Hall and M. Suzuki, Explicit R-parity breaking in supersymmetric models, Nucl. Phys. B 231 (1984) 419 [SPIRES]. 
[26] S. Dawson, R-parity breaking in supersymmetric theories, Nucl. Phys. B 261 (1985) 297 [SPIRES].

[27] R. Barbier et al., R-parity violating supersymmetry, Phys. Rept. 420 (2005) 1 [hep-ph/0406039] [SPIRES].

[28] D. Choudhury and S. Raychaudhuri, R-parity violation at HERA?, Phys. Lett. B 401 (1997) 54 [hep-ph/9702392] [SPIRES].

[29] G. Altarelli, J.R. Ellis, G.F. Giudice, S. Lola and M.L. Mangano, Pursuing interpretations of the HERA large $Q^{2}$ data, Nucl. Phys. B 506 (1997) 3 [hep-ph/9703276] [SPIRES].

[30] E.L. Berger, B.W. Harris and Z. Sullivan, Single-top-squark production via R-parity-violating supersymmetric couplings in hadron collisions, Phys. Rev. Lett. 83 (1999) 4472 [hep-ph/9903549] [SPIRES].

[31] E.L. Berger, B.W. Harris and Z. Sullivan, Direct probes of R-parity violating supersymmetric couplings via single top squark production, Phys. Rev. D 63 (2001) 115001 [hep-ph/0012184] [SPIRES].

[32] W. Buchmüller, L. Covi, K. Hamaguchi, A. Ibarra and T. Yanagida, Gravitino dark matter in R-parity breaking vacua, JHEP 03 (2007) 037 [hep-ph/0702184] [SPIRES].

[33] S. Lola, P. Osland and A.R. Raklev, Radiative gravitino decays from R-parity violation, Phys. Lett. B 656 (2007) 83 [arXiv:0707.2510] [SPIRES].

[34] N.E. Bomark, S. Lola, P. Osland and A.R. Raklev, Gravitino dark matter and the flavour structure of R-violating operators, Phys. Lett. B 677 (2009) 62 [arXiv:0811.2969] [SPIRES].

[35] F. Takayama and M. Yamaguchi, Gravitino dark matter without R-parity, Phys. Lett. B 485 (2000) 388 [hep-ph/0005214] [SPIRES].

[36] G. Moreau and M. Chemtob, R-parity violation and the cosmological gravitino problem, Phys. Rev. D 65 (2002) 024033 [hep-ph/0107286] [SPIRES].

[37] V. Ben-Hamo and Y. Nir, Implications of horizontal symmetries on baryon number violation in supersymmetric models, Phys. Lett. B 339 (1994) 77 [hep-ph/9408315] [SPIRES].

[38] A.H. Chamseddine and H.K. Dreiner, Anomaly free gauged $R$ symmetry in local supersymmetry, Nucl. Phys. B 458 (1996) 65 [hep-ph/9504337] [SPIRES].

[39] P. Binetruy, S. Lavignac and P. Ramond, Yukawa textures with an anomalous horizontal Abelian symmetry, Nucl. Phys. B 477 (1996) 353 [hep-ph/9601243] [SPIRES].

[40] G. Bhattacharyya, Non-Abelian flavour symmetry and R-parity, Phys. Rev. D 57 (1998) 3944 [hep-ph/9707297] [SPIRES].

[41] P. Binetruy, E. Dudas, S. Lavignac and C.A. Savoy, Quark flavour conserving violations of the lepton number, Phys. Lett. B 422 (1998) 171 [hep-ph/9711517] [SPIRES].

[42] J.R. Ellis, S. Lola and G.G. Ross, Hierarchies of R-violating interactions from family symmetries, Nucl. Phys. B 526 (1998) 115 [hep-ph/9803308] [SPIRES].

[43] B.C. Allanach et al., The Snowmass points and slopes: benchmarks for SUSY searches, in Proceedings of the APS/DPF/DPB Summer Study on the Future of Particle Physics (Snowmass 2001), N. Graf ed., Snowmass U.S.A. (2001), pg. P125 [Eur. Phys. J. C 25] (2002) 113 [hep-ph/0202233] [SPIRES].

[44] WMAP collaboration, C.L. Bennett et al., First year Wilkinson Microwave Anisotropy Probe (WMAP) observations: preliminary maps and basic results, Astrophys. J. Suppl. 148 (2003) 1 [astro-ph/0302207] [SPIRES]. 
[45] WMAP collaboration, D.N. Spergel et al., First year Wilkinson Microwave Anisotropy Probe (WMAP) observations: determination of cosmological parameters,

Astrophys. J. Suppl. 148 (2003) 175 [astro-ph/0302209] [SPIRES].

[46] WMAP collaboration, D.N. Spergel et al., Wilkinson Microwave Anisotropy Probe (WMAP) three year results: implications for cosmology, Astrophys. J. Suppl. 170 (2007) 377 [astro-ph/0603449] [SPIRES].

[47] H.K. Dreiner and G.G. Ross, R-parity violation at hadron colliders, Nucl. Phys. B 365 (1991) 597 [SPIRES].

[48] F. de Campos et al., Probing bilinear R-parity violating supergravity at the LHC, JHEP 05 (2008) 048 [arXiv:0712.2156] [SPIRES].

[49] F. de Campos et al., Probing neutrino mass with displaced vertices at the Tevatron, Phys. Rev. D 71 (2005) 075001 [hep-ph/0501153] [SPIRES].

[50] D. Choudhury and P. Roy, New constraints on lepton nonconserving R-parity violating couplings, Phys. Lett. B 378 (1996) 153 [hep-ph/9603363] [SPIRES].

[51] H.K. Dreiner, M. Krämer and B. O'Leary, Bounds on R-parity violation from leptonic and semi-leptonic meson decays, Phys. Rev. D 75 (2007) 114016 [hep-ph/0612278] [SPIRES].

[52] Y. Kao and T. Takeuchi, Constraints on R-parity violation from recent Belle/Babar data, arXiv:0909.0042 [SPIRES].

[53] R. Bose, Rare $\tau$ decays in R-parity violating supersymmetry, J. Phys. G 38 (2011) 065003 [arXiv: 1012.1736] [SPIRES].

[54] T. Sjöstrand, S. Mrenna and P.Z. Skands, A brief introduction to PYTHIA 8.1, Comput. Phys. Commun. 178 (2008) 852 [arXiv:0710.3820] [SPIRES].

[55] B.C. Allanach et al., SUSY Les Houches accord 2, Comp. Phys. Commun. 180 (2009) 8 [arXiv:0801.0045] [SPIRES].

[56] B.C. Allanach, SOFTSUSY: a C++ program for calculating supersymmetric spectra, Comput. Phys. Commun. 143 (2002) 305 [hep-ph/0104145] [SPIRES].

[57] T. Sjöstrand, S. Mrenna and P.Z. Skands, PYTHIA 6.4 physics and manual, JHEP 05 (2006) 026 [hep-ph/0603175] [SPIRES].

[58] M. Cacciari and G.P. Salam, Dispelling the $N^{3}$ myth for the $k_{t}$ jet-finder, Phys. Lett. B 641 (2006) 57 [hep-ph/0512210] [SPIRES].

[59] S.D. Ellis and D.E. Soper, Successive combination jet algorithm for hadron collisions, Phys. Rev. D 48 (1993) 3160 [hep-ph/9305266] [SPIRES].

[60] S. Catani, Y.L. Dokshitzer, M.H. Seymour and B.R. Webber, Longitudinally invariant $k_{t}$ clustering algorithms for hadron hadron collisions, Nucl. Phys. B 406 (1993) 187 [SPIRES].

[61] Particle Data Group collaboration, K. Nakamura et al., Review of particle physics, J. Phys. G 37 (2010) 075021 [SPIRES].

[62] ATLAS collaboration, ATLAS detector and physics performance technical design report, volume 1, CERN-LHCC-99-014, CERN, Geneva Switzerland (1999) [ATLAS-TDR-14] [SPIRES].

[63] ATLAS collaboration, ATLAS detector and physics performance technical design report, volume 2, CERN-LHCC-99-015, CERN, Geneva Switzerland (1999) [ATLAS-TDR-15] [SPIRES]. 
[64] H. Baer, C.-H. Chen and X. Tata, Impact of hadronic decays of the lightest neutralino on the reach of the CERN LHC, Phys. Rev. D 55 (1997) 1466 [hep-ph/9608221] [SPIRES].

[65] D. Choudhury, M. Datta and M. Maity, Looking for the top-squark at the Tevatron with four jets, Phys. Rev. D 73 (2006) 055013 [hep-ph/0508009] [SPIRES].

[66] J.M. Butterworth, J.R. Ellis, A.R. Raklev and G.P. Salam, Discovering baryon-number violating neutralino decays at the LHC, Phys. Rev. Lett. 103 (2009) 241803 [arXiv:0906.0728] [SPIRES].

[67] S. Kraml and A.R. Raklev, Same-sign top quarks as signature of light stops at the LHC, Phys. Rev. D 73 (2006) 075002 [hep-ph/0512284] [SPIRES].

[68] See e.g. P. Binetruy, Supersymmetric extensions of the standard model, plenary talk at the International Europhysics Conference on High-Energy Physics, Jerusalem Israel August 19-26 1997 [SPIRES].

[69] S. Lola and G.G. Ross, Neutrino masses from U(1) symmetries and the super-Kamiokande data, Nucl. Phys. B 553 (1999) 81 [hep-ph/9902283] [SPIRES].

[70] CMS collaboration, The CMS physics technical design report, volume 1, CERN-LHCC-2006-001, CERN, Geneva Switzerland (2006) [CMS-TDR-008-1] [SPIRES].

[71] CMS collaboration, The CMS physics technical design report, volume 2, CERN-LHCC-2006-021, CERN, Geneva Switzerland (2006) [CMS-TDR-008-2] [J. Phys. G 34 (2007) 995] [SPIRES].

[72] H. Baer, C.-H. Chen, F. Paige and X. Tata, Signals for minimal supergravity at the CERN Large Hadron Collider II: multilepton channels, Phys. Rev. D 53 (1996) 6241 [hep-ph/9512383] [SPIRES].

[73] I. Hinchliffe, F.E. Paige, M.D. Shapiro, J. Soderqvist and W. Yao, Precision SUSY measurements at CERN LHC, Phys. Rev. D 55 (1997) 5520 [hep-ph/9610544] [SPIRES].

[74] I. Hinchliffe et al., Precision SUSY measurements at LHC: point 3, LBNL-40954, Lawrence Berkeley National Lab, Berkeley U.S.A. (1998) [SPIRES]. .

[75] H. Bachacou, I. Hinchliffe and F.E. Paige, Measurements of masses in SUGRA models at CERN LHC, Phys. Rev. D 62 (2000) 015009 [hep-ph/9907518] [SPIRES].

[76] B.C. Allanach, C.G. Lester, M.A. Parker and B.R. Webber, Measuring sparticle masses in non-universal string inspired models at the LHC, JHEP 09 (2000) 004 [hep-ph/0007009] [SPIRES].

[77] B.K. Gjelsten, D.J. Miller, 2 and P. Osland, Measurement of SUSY masses via cascade decays for SPS 1a, JHEP 12 (2004) 003 [hep-ph/0410303] [SPIRES].

[78] D. Aristizabal Sierra, M. Hirsch and W. Porod, R-parity violating sneutrino decays, JHEP 09 (2005) 033 [hep-ph/0409241] [SPIRES].

[79] B. Mukhopadhyaya, S. Roy and F. Vissani, Correlation between neutrino oscillations and collider signals of supersymmetry in an R-parity violating model, Phys. Lett. B 443 (1998) 191 [hep-ph/9808265] [SPIRES].

[80] W. Porod, M. Hirsch, J. Romao and J.W.F. Valle, Testing neutrino mixing at future collider experiments, Phys. Rev. D 63 (2001) 115004 [hep-ph/0011248] [SPIRES]. 